\title{
Analysis of Gene Expression from Systemic Lupus Erythematosus Synovium Reveals a Profile of Activated Immune Cells and Inflammatory Pathways
}

Erika L. Hubbard ${ }^{\mathrm{a},{ }^{*}}$, Michelle D. Catalina ${ }^{\mathrm{a}, \mathrm{b}}$, Sarah Heuer ${ }^{\mathrm{a}, \mathrm{c}}$, Prathyusha Bachali ${ }^{\mathrm{a}}$, Robert Robl ${ }^{\mathrm{a}}$, Nicholas S. Geraci ${ }^{\mathrm{a}, \mathrm{d}}$, Amrie C. Grammer ${ }^{\mathrm{a}, \#}$, Peter E. Lipsky ${ }^{\mathrm{a}, \#}$

${ }^{a}$ RILITE Research Institute and AMPEL BioSolutions LLC; 250 West Main Street Suite \#300 Charlottesville, VA 22902, USA.

*Address correspondence to erika.hubbard@ampelbiosolutions.com; 250 West Main Street Suite 300, Charlottesville, VA 22902, USA.

${ }^{\mathrm{b}}$ Present address: EMD Serono Research \& Development Institute, 45 A Middlesex Turnpike Billerica, MA 01821

${ }^{c}$ Present address: The Jackson Laboratory, Tufts Graduate School of Biomedical Sciences, 600

Main Street Bar Harbor, ME 04609, USA

${ }^{\mathrm{d}}$ Present address: Profiler Business Unit, Genedata Inc, 1 Cranberry Hill, Lexington, MA 02421, USA

${ }^{\#}$ Contributed equally to this work 


\begin{abstract}
Arthritis is a common manifestation of systemic lupus erythematosus (SLE) yet understanding of the underlying pathogenic mechanisms remains incomplete. We, therefore, interrogated gene expression profiles of SLE synovium to gain insight into the nature of lupus arthritis (LA), using osteoarthritis (OA) and rheumatoid arthritis (RA) as comparators. Knee synovia from SLE, OA, and RA patients were analyzed for differentially expressed genes (DEGs) and also by Weighted Gene Co-expression Network Analysis (WGCNA) to identify modules of highly co-expressed genes. Genes upregulated and/or co-expressed in LA revealed numerous immune/inflammatory cells dominated by a myeloid phenotype, whereas OA was characteristic of fibroblasts and RA of T- and B-cells. Upstream regulator analysis identified CD4OL and inflammatory cytokines as drivers of the LA gene expression profile. Genes governing trafficking of immune cells into the synovium by chemokines were identified, but not in situ generation of germinal centers. GSVA confirmed activation of specific myeloid and lymphoid cell types in LA. Numerous therapies were predicted to target LA, including TNF, NFkB, MAPK, and CDK inhibitors. Detailed gene expression analysis identified a unique pattern of cellular components and physiologic pathways operative in LA, as well as drugs potentially able to target this common manifestation of SLE.
\end{abstract}

Keywords: SLE; arthritis; synovium; gene expression 


\section{INTRODUCTION}

Systemic lupus erythematosus (SLE) is a complex autoimmune disease in which loss of self-tolerance gives rise to pathogenic autoantibodies causing widespread inflammation and tissue damage [1]. Lupus arthritis (LA) is a common manifestation of SLE with 65 to $95 \%$ of lupus patients reporting joint involvement during the course of their disease [2].

Despite the high frequency of LA, an understanding of the underlying pathogenic mechanisms remains incomplete. Cytokines, such as IL-6, and anti-dsDNA autoantibodies are thought to play a role [3-5]. Other autoantibodies including anti-ribonucleoprotein, anti-histone, and anti-proliferating cell nuclear antigen have been implicated in LA along with evidence of increased C-reactive protein (CRP) and erythrocyte sedimentation rate (ESR) $[4,6,7]$.

The lack of a better understanding of the nature of LA relates to the difficulty of obtaining tissue samples and the absence of relevant and reliable animal models. Despite this, in most recent clinical trials of potential lupus therapies, arthritis is a principal manifestation and the success of a tested therapy can depend on its ability to suppress synovial inflammation. Therefore, it is essential to understand more about the pathogenic mechanisms operative in LA.

One way to evaluate the pathologic processes involved in LA is to analyze gene expression profiles in the affected synovium. Previous work analyzed global gene expression profiles and histology of SLE, rheumatoid arthritis (RA), and osteoarthritis (OA) synovium [8-9] to begin to elucidate the inflammatory mechanisms in each disease and focused on the type 1 interferon pathway in LA. Here, we expand upon these studies by applying contemporary bioinformatic techniques to assess the only gene expression data set available to gain additional insight into the pathogenesis of LA. Using a multipronged, bioinformatic and systems biology 
approach, we provide an expanded view of SLE synovitis that might serve as the basis to identify new targeted therapies.

\section{RESULTS}

\section{Bioinformatic and Pathway Analysis of LA and OA Gene Expression}

Differential expression (DE) analysis demonstrated 6,496 differentially expressed genes (DEGs) in LA versus OA (Fig. 1a), of which 2,477 transcripts were upregulated whereas 4,019 transcripts were downregulated. The upregulated DEGs included 243 immune cell-specific transcripts (odds ratio of 2.84, $\mathrm{p}<2.2 \mathrm{e}-16$, Fisher's Exact Test), indicating a significant immune/inflammatory cell infiltrate. There was considerable enrichment of T-cell, B-cell, plasma-cell, and myeloid-cell transcripts among the upregulated DEGs (Fig. 1b), whereas fibroblast-associated genes were increased in OA. Immune signaling and immune cell surface markers, as well as the interferon transcriptional program, pattern recognition receptors (PRRs), and MHC Class I and II were enriched in LA, along with intracellular signaling, apoptosis, the proteasome, and processing/packaging material inside cells.

Of the 4,019 downregulated DEGs, only 17 were immune cell transcripts and thus downregulated genes did not reflect a change in hematopoietic cells (odds ratio of 0.0749, p=1). Notably, however, the fibroblast gene signature was downregulated (Fig. 1c). Functional analysis identified several molecular processes that were decreased in LA, most of which related to transcriptional activity/nuclear processes and cytoskeletal and integrin pathway changes. A list of genes significantly up- and downregulated in LA can be found in Supplementary Data S1 online.

Ingenuity Pathway Analysis (IPA) identified predominantly innate immune signaling processes, including a proinflammatory macrophage response, interferon signaling and 
inflammasome pathway activation, as well as an adaptive immune response (e.g., Th1 Pathway) (Fig. 2a). These were confirmed by functional analysis of IPA-predicted upstream regulators (UPRs) of the disordered gene expression profiles in LA (Fig. 2b), which identified specific intracellular signaling molecules, PRRs, and secreted immune proteins, including type 1 and 2 interferons. Of note, a number of pro-apoptotic genes, including TNF, TNFSF10, and FAS were predicted UPRs. The full export of IPA canonical pathway and upstream regulator data can be found in Supplementary Data S3-6 online.

To confirm DEG-identified molecular pathways and further interrogate LA gene expression, we implemented weighted gene co-expression network analysis (WGCNA), which serves as an orthogonal bioinformatic approach. WGCNA identified 52 modules, six of which were highly co-expressed and significantly associated with LA (see Supplementary Figs. S1, S2, and Supplementary Table S1 online). Of the six modules with significant, positive correlations to features of lupus, some correlated with the presence of LA and some modules correlated with SLEDAI and anti-dsDNA. The associations of the WGCNA modules with features of LA can be seen in Supplementary Figs. S1, S3, S4, and Supplementary Data S7-13 online. Many of the modules contained immune/inflammatory cell signatures. Notably, plasma cell genes were found in the midnightblue module and included $\operatorname{IgG} 1$, $\operatorname{IgM}$, and $\operatorname{IgD}$, indicating both pre- and postswitch plasmablasts/plasma cells as well as the presence of $\operatorname{Ig} \kappa, \operatorname{Ig} \lambda$, and numerous VL chains, signifying a polyclonal population (see Supplementary Fig. S5 online). Midnightblue, however, was upregulated in two of four lupus patients and negatively correlated with systemic disease (see Supplementary Fig. S1 online). The blue module, which was negatively correlated with LA, was enriched in synovial fibroblasts (see Supplementary Fig. S4, Supplementary Table S2 
online), whereas the brown module, which was positively associated with LA, was less likely to be enriched for synovial fibroblasts (see Supplementary Fig. S3 online).

WGCNA modules were enriched in immune/inflammatory processes, including interferon stimulation, antigen presentation, immune cell surface markers, immune signaling, pathogenic pattern recognition, and lysosome function (see Supplementary Fig. S6 online), confirming BIG-C and IPA results. Further IPA canonical pathway analysis, IPA UPR analysis, and STRING/MCODE clustering of WGCNA modules (see Supplementary Fig. S7 online) indicated that the brown and navajowhite 2 modules were characterized by myeloid cell responses, whereas the honeydew1 module was most characterized by interferon signaling and the darkgrey module with cellular activation, antigen presentation, and proinflammatory signaling. In contrast, the midnightblue module revealed T-cell: B-cell crosstalk, T-cell activation and differentiation, and B-cell signaling. Finally, salmon4 was not enriched in immune cells (see Supplementary Fig. S3 online).

\section{Lymphocyte Trafficking and GC Activity in LA}

We assessed chemokine receptor-ligand pairs and adhesion molecules to understand mechanisms of immune/inflammatory cell localization in LA. Numerous chemokine receptorligand pairs were expressed in SLE synovium, including CCR5-CCL4/5/8, CCR1-CCL5/7/8/23, and CXCR6-CXCL16 (Table 1). Of note, CXCL13 was expressed in the midnightblue module and upregulated, although its receptor $C X C R 5$ was not detected in LA-associated WGCNA modules nor in DEGs. Adhesion molecules were found in LA-associated WGCNA modules including VCAM1, CD44, CADM3, and ITGB2.

We also examined expression of specific follicular helper $\mathrm{T}$ cell $\left(\mathrm{T}_{\mathrm{fh}}\right)$ and germinal center (GC) B-cell markers to determine whether GCs might contribute to LA pathogenesis (see 
Supplementary Fig. S8 online). ICOS, but not other $\mathrm{T}_{\mathrm{fh}}$ markers, were found in LA. In addition, several GC B-cell markers were upregulated in SLE synovium, including, CXCL13 and IRF4. However, BCL6 and RGS16 were notably downregulated and RGS13 was not differentially expressed between SLE and OA. A cluster of GC B-cell markers that tended to be upregulated were co-expressed in the midnightblue module.

\section{GSVA Enrichment of Immune and Tissue Populations and Signaling Pathways}

To assess the differences between SLE and OA synovitis on an individual sample basis, GSVA of various informative gene sets was carried out (Fig. 3, see Supplementary Data S14 online). Enrichment of hematopoietic cell types confirmed the presence of an immune infiltrate in LA, but not OA (Fig. 3a). Most cell types, including lymphoid and myeloid populations, were enriched. Cytokine signaling was also enriched in LA, including both proinflammatory cytokine signaling as well as inhibitory cytokines (Fig. 3b). Of note, the downstream signature induced by TNF signaling was significantly enriched in LA ( $\mathrm{p}=0.00918)$. Whereas antigen presentation markers, cellular activation markers, and the inflammasome pathway were enriched in LA compared to OA, a cell cycle/proliferation signature and complement pathways were not enriched (Fig. 3c). Along with upregulation of inhibitory cytokines, inhibitory receptors and negative regulation of T cells were enriched in LA (Fig. 3d). Most of the previously noted IPApredicted canonical signaling pathways were enriched in SLE synovium aside from signaling by the eukaryotic initiation factor eIF2 (Fig. 3e).

Conversely, OA synovium was enriched in tissue repair/destruction and markers of fibroblasts (Fig. 4a). Querying LA and OA synovial expression profiles with the co-expressed genes from fibroblast subpopulations described in human RA and OA synovium [10], we found that two resident synovial sublining fibroblast populations, $\mathrm{CD} 34^{+}$and $\mathrm{DKK} 3^{+}$, were 
significantly increased in OA over LA (Fig. 4b; $\mathrm{p}=0.00148$ and 0.00213 , respectively). However, co-expressed genes characterizing the HLA-DR ${ }^{\text {hi }}$ sublining fibroblast population were significantly enriched in LA ( $\mathrm{p}=2.70 \mathrm{e}-05)$. Interestingly, using the same approach to assess macrophage populations also described in human RA and OA [10-11], we found quiescent macrophages and IFN-activated macrophages significantly increased in LA (Fig. 4c; p=0.0332 and $1.14 \mathrm{e}-06$, respectively), whereas phagocytic macrophages were associated with OA. $\mathrm{HBEGF}^{+}$proinflammatory macrophages tended to be enriched in LA but did not reach statistical significance $(\mathrm{p}=0.0993)$.

Other unique macrophage subpopulations have been described in mouse synovium, including a CX3CR $1^{+}$resident subtype resembling epithelial cells [12]. Taking the co-expressed genes of the human orthologs of this macrophage signature and others detected in healthy mouse synovium, we found that two types of interstitial macrophages, including $\mathrm{MHCII}^{+}$and RELM- $\alpha^{+}$ populations, were significantly more abundant in LA ( $\mathrm{p}=0.00549$ and 0.00802 , respectively) as well as a group of $\mathrm{ACP5}^{+}$osteoclast precursors (Fig. 4d; p=9.37e-04). The CX3CR1 ${ }^{+}$lining macrophage population from healthy mice was not significantly enriched in either LA or OA synovium ( $\mathrm{p}=0.110)$; however, the $\mathrm{CX} 3 \mathrm{CR} 1^{+}$lining macrophage population from murine inflammatory arthritis were increased in LA (Fig. 4e). Additionally, inflammatory arthritisassociated $\mathrm{MHCII}^{+}$and RELM- $\alpha^{+}$interstitial macrophages were enriched in LA as were MHCII ${ }^{\mathrm{hi}}$ dendritic cells and $\mathrm{CCR} 2^{+} \mathrm{IL}_{1 \mathrm{~B}}{ }^{+}$monocyte-derived macrophages.

\section{Comparison of Gene Expression in LA and RA Synovitis}

Fewer genes were upregulated in RA, but $18 \%$ of these genes identified immune/inflammatory cells compared with $10 \%$ of genes upregulated in LA (Fig. 5a). Characterization of upregulated DEGs by cell signatures revealed greater numbers of myeloid 
and monocyte/macrophage-specific transcripts in LA compared to RA, whereas immune infiltrates in RA were more characteristic of T- and B-cells. B-cells, naïve/memory cells, and gamma delta (gd) T cells were significantly increased in RA over LA ( $p=0.0249, p=0.0121$, and $\mathrm{p}=0.00689$, respectively), whereas monocytes/macrophages, inhibitory macrophages, and M2 macrophages were significantly increased in LA over RA (Fig. 5b; $p=0.0160, p=0.00306$, and $\mathrm{p}=0.00719$, respectively).

Interferon signaling pathways, the complement pathway, inflammasome pathway, antigen presentation, inhibitory cytokines, and inhibitory receptors were substantially enriched in LA, whereas negative regulation of T-cells and cell cycle tended to be enriched in RA (Fig. 5ce). Downstream signatures of TNF, IL-1, and IL-6 tended to be enriched in LA compared to RA. Finally, other pathways, including response to stimuli, phagocytosis, chemokine signaling, BCR signaling, and PI3K signaling, were considerably enriched in LA (Fig. 5f).

Transcripts associated with tissue repair/destruction were significantly enriched in RA (see Supplementary Fig. S9 online; p=9.95e-04). Enrichment of general fibroblast markers was not uniform in either tissue. Nonetheless, HLA-DR ${ }^{\text {hi }}$ sublining and CD55 ${ }^{+}$lining fibroblasts were significantly associated with LA $\left(\mathrm{p}=0.00247\right.$ and $\mathrm{p}=1.39 \mathrm{e}-04$, respectively), whereas the CD34 ${ }^{+}$ and $\mathrm{DKK} 3^{+}$sublining populations were depleted in LA compared to RA.

$\mathrm{HBEGF}^{+}$proinflammatory macrophages and phagocytic macrophages tended to be more enriched in RA patients, although not uniformly, whereas quiescent and IFN-activated macrophages were more enriched in LA, the latter population reaching statistical significance (see Supplementary Fig. S9 online; p=0.00857) [10-11]. When detected, the normal and arthritis murine equivalent macrophage populations trended towards enrichment in LA, including the $\mathrm{CX} 3 \mathrm{CR} 1^{+}$lining macrophages [12]. 


\section{Compounds Predicted to Target LA}

Drugs predicted to reverse the abnormal gene expression profile of LA were identified by connectivity mapping to the LINCS database and are shown in Table 2 and Supplementary Table S3 online. Most abundantly predicted compounds include anti-cancer drugs targeting tubulin polymerization, MAPK signaling, and EGFR signaling, as well as current lupus standard-of-care therapies, including corticosteroids and NSAIDs/prostaglandin synthesis inhibitors. Interestingly, a few alternative medicines were predicted to counteract LA, including curcumin, capsaicin, resveratrol, and caffeine. In addition to the LINCS-predicted compounds, we sought to expand the potential list of therapies to include those targeting biological upstream regulators (BURs). The top 50 BURs determined by connectivity scoring with gene expression generated by knock down or overexpression studies in cell lines are summarized in Fig. 6a along with drugs that could potentially directly target these BURs, including ESR1, type 1 IFN, bromodomain and casein kinase inhibitors. Finally, the UPRs predicted by IPA were also matched with potential targeting drugs (Fig. 6b). Notably, 26\% of drugs targeting IPA upstream regulators were also predicted by LINCS BURs drug-target matches (see Supplementary Data S15-16 online), and included inhibitors of TNF, type I interferon, the NFkB pathway, JAK, and CDK.

\section{DISCUSSION}

Analyses of DEGs revealed a uniform inflammatory infiltrate in LA of mostly myeloid lineage cell types, including monocytes, M1 macrophages, antigen presenting cells, and other myeloid and hematopoietic cells. WGCNA indicated enrichment of other immune cell types, including activated and effector T cells, NK cells, B cells, plasma cells/plasmablasts, and both M1 and M2-polarized macrophages, suggesting both innate and adaptive mechanisms at play in 
LA, although the involvement of adaptive immune cells was less uniform than that of the innate immune system.

Our findings indicated that myeloid-lineage cells were enriched in LA and, therefore, may play a central role in the observed inflammation. IPA revealed monocyte/macrophagemediated phagocytosis and NO and ROS production signaling pathways, and GSVA confirmed gene expression profiles of both inflammatory M1 and inhibitory M2 macrophages in LA. This aligns with prior histology suggesting the presence of infiltrating macrophages [9] and recent analysis of myeloid cells in SLE blood associated an M1 inflammatory phenotype with active versus inactive disease [13]. By comparison to single-cell transcriptional profiles from sorted murine synovial $\mathrm{CD}_{4} 5^{+} \mathrm{Cd} 11 \mathrm{~b}^{+} \mathrm{Ly}_{6 \mathrm{G}}^{-}$cells [12], significant enrichment of resident macrophage populations as well as non-resident infiltrating populations were identified, as well as antiinflammatory macrophage subpopulations and inhibitory and inflammatory cytokines, indicating that multiple macrophage subtypes and their secreted products may contribute to and perhaps protect against LA pathogenesis.

Fibroblast-unique genes were downregulated in LA, possibly representing local relative loss or diminished/altered function of resident fibroblasts. Pathologic fibroblast populations, potentially contributing to local tissue damage, have been shown to reside in the synovium of patients with leukocyte-rich RA [14-16], including a subpopulation of CD34 ${ }^{+}$sublining fibroblasts, which was decreased in LA. Another fibroblast population enriched in leukocyte-rich RA and characterized by higher expression of MHC Class II genes IL6 and CXCL12 appeared to also be enriched in LA [10]. However, this population was mainly characterized by interferon stimulated and MHC Class I/II genes, and, therefore, this signature cannot definitively be attributed to fibroblasts (see Supplementary Fig. S10 online). Furthermore, comparison of SLE 
and RA synovial gene profiles indicated maintenance of a lining CD55 ${ }^{+}$fibroblast layer in LA and tissue repair/destruction mechanisms in RA. LA may, therefore, differ from RA and OA, in which joint organ pathology is characterized by fibroblast-mediated tissue damage and, rather, be characterized by a loss of function or dysregulation of proinflammatory fibroblasts with destructive potential.

LA may also differentiate from RA in its immune cellularity and composition. A greater number of genes were found significantly altered in LA than in RA but a smaller portion of these transcripts could be attributed to immune/inflammatory cell populations, indicating an overall greater immune infiltrate in RA than in LA. Of the immune/inflammatory cell-specific transcripts identified, RA upregulated DEGs indicated increased T-cells, B-cells, NK/NKT-cells, and other lymphocytes, whereas LA upregulated DEGs were more characteristic of monocytes/macrophages and myeloid cells. Thus, LA may be more myeloid-mediated than RA. GSVA replicated this finding with significant upregulation of the core type I interferon signature, antigen presentation signature, inflammasome pathways, and monocyte/macrophage cell populations in LA including, notably, more inhibitors of inflammation. Specific macrophage and dendritic cell subsets originally identified in mouse synovium [12] were also more enriched in LA. Interestingly, the downstream TNF, IL-1, and IL-6 signatures tended to be more enriched in LA than RA, indicating potential for repurposing anti-TNF biologics, the IL-1 antagonists anakinra and canakinumab, and IL-6R antagonist tocilizumab to treat LA.

The detection of Ig heavy chain pre- and post-switch plasma cells in LA was notable. IRF4, XBP1, and PRDM1, genes essential for plasma cell maturation [17], were all detected in LA. There was some evidence of GC formation in LA, but BCL6, AICDA, and RGS13 were not upregulated nor detected in an LA-associated WGCNA module. However, CXCL13, encoding a 
chemoattractant that has been reported in RA synovial GCs [18], was strongly upregulated. These findings suggest that fully-developed GCs are not a routine part of LA. Rather, it is more likely that lupus synovium contains lymphoid aggregates that support B-cell proliferation and autoantibody formation, as reported in the spleen in immune thrombocytopenia [19]. An interesting caveat to our data is the strong negative correlations of the midnightblue module, which contains the plasma cell signature, to SLEDAI and anti-dsDNA, whilst being positively correlated to LA. This suggests that the presence of plasmablasts/plasma cells in lupus synovium may not contribute significantly to systemic autoantibody levels and extra-articular lupus disease activity. Rather, the nature of the local inflammation may facilitate entry of circulating plasmablasts/plasma cells into the synovial space and/or their local differentiation.

The overexpression of numerous chemokines and chemokine receptors suggests chemokine signaling may play an important role in the infiltration of immune/inflammatory cells into lupus synovium. CXCR3 and its ligands CXCL9, CXCL10, and CXCL11 were all found upregulated and co-expressed in the midnightblue module, which contained a robust lymphocyte signature. This signaling axis is known to be induced by IFN $\gamma$ and is involved in the recruitment of activated lymphocytes, particularly of naïve T cells and their differentiation into Th1 cells [20]. CXCR3 and CXCR4, both of which were upregulated in LA, are additionally important for the homing and maintenance of plasma cells [21]. These chemokine receptors could be involved in the recruitment of circulating plasmablasts/plasma cells into lupus synovium and/or their in situ retention and/or differentiation [22-24]. Other chemokines and their receptors such as CCR5CCLA/CCL5 could contribute to recruitment of other leukocytes into the synovium, including macrophages, monocytes, and T-cells [25]. 
We utilized gene expression analysis to predict novel drugs that might target abnormally expressed genes or pathways and suppress inflammation. Predicted drugs and compounds identified novel potential therapies, but also confirmed current treatments by identifying standard-of-care lupus drugs such as glucocorticoids, methotrexate, aspirin, and cyclosporine. Notably, a large number of anti-cancer drugs with variable mechanisms of action were also predicted.

Drugs targeting the CDK family were predicted to revert the LA gene signature and may point to potential repurposing of drugs such as palbociclib or related seliciclib and other CDK inhibitors that have been shown to ameliorate nephritis in animal models [26], possibly by reducing proliferation of lupus T- and B-cells in vitro [27]. Similarly, bucladesine was one of nine phosphodiesterase inhibitors predicted to suppress LA. Other novel druggable therapeutic targets include ROCK2, SYK, PARP1 and PARP2, and HDAC.

Notably, a large number of sodium channel blockers were predicted to target LA, possibly related to increased nervous innervation of the inflamed synovium. Neurologic targets included the acetylcholine, dopamine, serotonin, GABA-A, adrenergic, and glutamate receptors. These may have been predicted based on changes in the innervation of the inflamed tissue, although an effect on immune/inflammatory cells is also possible [28]. Similarly, transmembrane ion channels were predicted targets of the LA gene program and may reflect dysregulation of innervation or a role on immune/inflammatory cells.

Multiple drug predictions were made surrounding the estrogen and progesterone receptors. It is well-known that women are affected by systemic lupus 10 times more often than men and that sex hormones are involved in modulating the immune system [29]. While glucocorticoids have mainly anti-inflammatory and immunosuppressive effects, sex hormones 
such as estrogen and progesterone may have either pro-inflammatory or anti-inflammatory effects depending on the types of receptors expressed and other factors [30]. Estrogen may increase risk of disease by favoring autoreactive B-cells and promoting type I interferon production, whereas progesterone seems to counteract these effects [31]. Thus, the right balance of these hormones may attenuate disease activity. In an all-female cohort, we found ESRl, encoding the alpha estrogen receptor, to be an upstream regulator of LA. Tamoxifen was also repeatedly suggested as a potential therapy for LA and has shown utility in murine lupus and in human lupus T-cells [32-34]. However, no clinical trials of Tamoxifen have been conducted in lupus or other autoimmune disease and cases of Tamoxifen-induced lupus and other adverse outcomes have been reported [35-36]. Thus, it is clear that female hormone receptors are important in LA pathogenesis but delineating their specific roles and the crosstalk between glucocorticoids and sex hormones needs further study.

Bioinformatic analysis of LA revealed a pattern of immunopathogenesis in which myeloid cell-mediated inflammation dominates. The breadth of the immune response underlying LA provides a basis for multiple avenues of therapeutic intervention to be considered that mouse models and previous studies have not provided. With these findings we can begin to hypothesize specific candidate target genes and pathways from which to develop or repurpose drugs to treat and improve LA specifically.

\section{METHODS}

\section{Gene Expression Data Sourcing and Patient Characteristics}

Publicly available gene expression data from synovial biopsies from the knees of 4 SLE, 5 OA, and 7 RA subjects with active articular disease were obtained from NCBI Gene Expression 
Omnibus (GEO) under accession GSE36700 [8]. The SLE patients assessed had a mean ( \pm s.d.) SLEDAI of 8.25 (1.71), CRP of $12.5 \mathrm{mg} / \mathrm{L}$ (4.12), C3 of $82.5 \mathrm{mg} / \mathrm{dL}$ (28.0), C4 of $13 \mathrm{mg} / \mathrm{dL}$ (3.56) and anti-dsDNA of 97.6 IU/mL (77.0), and all patients had active arthritis at the time biopsy was taken. Complete patient data can be found in Supplementary Data S17 online. Data processing and analysis were conducted within the $\mathrm{R}$ statistical programming platform using relevant Bioconductor packages.

\section{Data Normalization}

All raw data files underwent background correction and GCRMA normalization resulting in log2 intensity values compiled into expression set objects (e-sets). Outliers were identified through the inspection of first, second, and third principal components and through inspection of array dendrograms calculated using Euclidean distances and clustered using average/UPGMA agglomeration. GSM899013_OA5 was consistently identified as an outlier and excluded from further analyses. Low intensity probes were removed by visual assignment of a 2.34 threshold cutoff upon a histogram of binned $\log 2$-transformed probe intensity values.

\section{Differential Gene Expression}

Identification of DEGs in SLE vs OA samples $(n=8)$ and RA vs OA samples $(n=11)$ was conducted using the LIMMA package in R. To increase the probability of finding DEGs, both Affy chip definition files (CDFs) and BrainArray CDFs were used to created and annotate e-sets, analyzed separately, then results merged. Linear models of normalized gene expression values were created through empirical Bayesian fitting. Resultant p-values were adjusted for multiple hypothesis testing using the Benjamini-Hochberg correction. Significant probes were filtered to retain a pre-specified False Discovery Rate $($ FDR $)<0.2$ and duplicate probes were removed 
again to retain the most significant probe. The FDR was assigned a priori to avoid excluding false negative probes. The full list of DEGs can be found in Supplementary Data S1-2 online.

\section{Weighted Gene Co-expression Network Analysis (WGCNA)}

The same normalized and filtered data (Affy CDFs only) were inputted into WGCNA to conduct an unsupervised clustering analysis yielding statistically co-expressed modules of genes used for further biological interrogation. A scale-free topology matrix (TOM) was calculated to encode the network strength between probes. TOM distances were used to cluster probes into WGCNA modules. Resulting co-expression networks were trimmed using dynamic tree cutting and the deepSplit function in R. Partitioning around medoids (PAM) was also utilized to assign outliers to the nearest cluster. Modules were given random color assignments and expression profiles summarized by a module eigengene (ME). Final membership of probes representing the same gene were decided based on strongest within-module correlation to the ME value. For each module, ME values were correlated by Pearson correlation to clinical data including cohort, SLEDAI, anti-dsDNA, C3, C4, and CRP levels. Cohort was represented as a binary variable where $\mathrm{SLE}=1$ and $\mathrm{OA}=0$ whereas the remaining clinical data were continuous variables. Full module gene lists can be found in Supplementary Data S8-13 online.

\section{Quality Control (QC) and Selection of WGCNA Modules}

WGCNA modules of interest underwent a QC process to ensure modules were reflective of disease state. First, ME expression per patient was visually inspected to assess consistency of gene expression in a given cohort. Second, module membership, or eigengene-based connectivity $\left(\mathrm{k}_{\mathrm{ME}}\right.$ ), was plotted against probe correlation to the primary clinical trait of interest (SLEDAI) to gauge how well a given module agreed to the clinical trait. Finally, the Pearson correlations of 
MEs to the clinical metadata were examined. Absolute values of correlation coefficients in the range $0.5-1$ were considered strong and alpha $=0.05$ determined significance.

\section{Functional Analysis}

Immune/Inflammation-Scope (I-Scope) and Biologically Informed Gene Clustering (BIG-C) are functional aggregation tools for characterizing immune cells by type and biologically classifying large groupings of genes, respectively. I-Scope categorizes gene transcripts into a possible 32 hematopoietic cell categories based on matching 926 transcripts known to mark various types of immune/inflammatory cells. BIG-C sorts genes into 52 different groups based on their most probable biological function and/or cellular/subcellular localization. Tissue-Scope (T-Scope) is an additional aggregation tool to characterize cell types found in specific tissues. In these analyses only the two T-Scope categories relevant to the synovium were used: fibroblasts and synoviocytes.

\section{Network Analysis}

Cytoscape (V3.6.1) software was used to visualize protein-protein interactions based on the Search Tool for the Retrieval of Interacting Genes/Proteins (STRING) database via the stringApp plugin application. A confidence score of 0.40 was used. The clustermaker2 plugin application was used to created MCODE clusters of interrelated genes using a network scoring degree cutoff of 2 , node score cutoff of 0.2 , maximum depth of 100, and k-Core of 2. Genes not recognized by the STRING database were removed from datasets prior to upload into Cytoscape.

\section{Ingenuity ${ }^{\circledR}$ Pathway Analysis (IPA)}

The canonical pathway and UPR functions of IPA core expression analysis (Qiagen) were used to interrogate DEGs and WGCNA module gene lists. Core expression analyses were based on fold change if uploaded genes were differentially expressed; otherwise, a fold change of one was 
used. Canonical pathways and UPRs were considered significant if $\mid$ Activation Z-Score $\mid \geq 2$ and overlap p-value $\leq 0.01$

\section{Gene Set Variation Analysis (GSVA)}

The GSVA R package was used as a non-parametric, unsupervised gene set enrichment method. Enrichment scores were calculated using a Kolgomorov Smirnoff (KS)-like random walk statistic to estimate variation of pre-defined gene sets. The inputs for the GSVA algorithm were e-sets containing $\log 2$ microarray expression values (Affy HGU133plus2 definitions) and predefined gene sets co-expressed in SLE datasets. Low-intensity probes were filtered out based on interquartile range (IQR) [37]. GSVA was conducted on the remaining network and Welch's ttest was used to detect significant differences in enrichment between cohorts at an alpha level of 0.05, followed by calculation of Hedge's g effect size with correction for small samples. Welch's t-test was used to account for unequal variances in both the SLE and OA populations and RA and OA populations.

Enrichment gene sets containing cell type- and process-specific genes listed in Supplementary Data S14 online were created through an iterative process of identifying DE transcripts pertaining to a restricted profile of hematopoietic cells in 13 SLE microarray datasets and checked for expression in purified T cells, B cells, and monocytes to remove transcripts indicative of multiple cell types. Genes were identified through literature mining, GO biological pathways, and STRING interactome analysis as belonging to specific categories [38]. Select gene sets (i.e., TNF-induced, IFN $\beta$-upregulated, M1 and M2) were derived directly from in vitro experiments [39-40]. The M1 signature was edited to remove interferon stimulated genes. Additionally, IL-1 and IL-6 gene sets were derived from the first three tiers of the respective PathCards signaling pathways. 


\section{Co-expression Analysis}

Co-expression analyses of literature-derived signatures published in mouse and human synovium were conducted in R. Briefly, Spearman's rank correlation coefficients and p-values were computed using the rcorr() function based upon input log2 expression values for each gene in each SLE and OA sample. Spearman correlations were chosen to avoid assuming linear relationships. The input gene signature was refined to contain genes significantly correlating with at least $25 \%$ of the original gene signature at an alpha level of 0.05 , then refined again to contain genes positively correlated with at least $25 \%$ of the new signature (i.e., Spearman's rho>0). The final co-expressed signatures were used as GSVA gene sets. Mouse to human ortholog conversion was done using the homologene R package.

\section{LINCS Drug-Target Prediction and Biological Upstream Regulator Analysis}

The Library of Integrated Network-Based Cellular Signatures (LINCS) perturbation database (http://data.lincscloud.org.s3.amazonaws.com/index.html) was queried using a DEG list of significantly up- and down-regulated genes from the SLE and OA samples. Comparisons were made based on LINCS-computed connectivity scores, where -100 describes a transcriptional program perfectly opposing the user-uploaded gene signature and 100 describes a transcriptional program perfectly representative of the user-uploaded gene signature.

\section{Drug-Target Matching}

In addition to the LINCS-predicted compounds, LINCS-predicted biological upstream regulators (BURs) and IPA-predicted UPRs were annotated with respective targeting drugs and compounds to elucidate potential useful therapies in lupus synovitis. Drugs targeting gene products of interest both directly and indirectly were sourced by IPA, the Connectivity Map via the drug repurposing tool, GeneCards, STITCH (V5.0), Combined Lupus Treatment Scoring (CoLTS)- 
scored drugs [41], FDA labels, DrugBank, literature mining, and queries of clinical trials databases. The CLUE drug repurposing tool was accessed at https://clue.io/repurposing-app.

\section{STITCH}

The Search Tool for Interactions of Chemicals (STITCH) (V5.0) database (http://stitch.embl.de/) of known and predicted protein-protein and protein-chemical interactions was used to predict direct and indirect drug targeting mechanisms. For each gene product of interest, the top 10 interactors were analyzed and drugs directly targeting the top interactors were matched according to the methods described. A medium confidence score cutoff of 0.4 for interaction predictions was used. Predicted interactions based solely on text-mining were not considered.

\section{Statistical Analysis}

Enrichment statistics in SLE vs OA were calculated by right-sided Fisher's Exact Test in R using the function fisher.test(). Statistical significance was obtained at $\mathrm{p}<0.05$.

\section{DATA AVAILABILITY}

The dataset analyzed during the current study is available in the NCBI GEO repository, https://www.ncbi.nlm.nih.gov/geo/query/acc.cgi?acc=GSE36700. Additional data generated from analyses are included in this published article (and its Supplementary Information files).

\section{REFERENCES}

1. Dörner, T., Jacobi, A. M. \& Lipsky, P. E. B cells in autoimmunity. Arthritis Research and Therapy 11, 247 (2009).

2. Grossman, J. Lupus arthritis. Best Pr. Res Clin Rheumatol 23, 495-506 (2009).

3. Ball, E., Gibson, D., Bell, A. \& Rooney, M. Plasma IL-6 levels correlate with clinical and 
ultrasound measures of arthritis in patients with systemic lupus erythematosus. Lupus 23, 46-56 (2013).

4. Eilertsen, G., Nikolaisen, C., Becker-Merok, A. \& Nossent, J. Interleukin-6 promotes arthritis and joint deformation in patients with systemic lupus erythematosus. Lupus 20, 607-613 (2011).

5. Illei, G. G. et al. Tocilizumab in systemic lupus erythematosus: Data on safety, preliminary efficacy, and impact on circulating plasma cells from an open-label phase I dosage-escalation study. Arthritis Rheum. 62, 542-552 (2010).

6. Hoffman, I. E. A. et al. Specific antinuclear antibodies are associated with clinical features in systemic lupus erythematosus. Ann. Rheum. Dis. 63, 1155-1158 (2004).

7. Cozzani, E., Drosera, M., Gasparini, G. \& Parodi, A. Serology of Lupus Erythematosus: Correlation between Immunopathological Features and Clinical Aspects. Autoimmune Dis. 2014, 321359 (2014).

8. Lauwerys, B. R. Gene expression profiles in synovial biopsies from patients with arthritis dataset. NCBI GEO. GSE36700 https://www.ncbi.nlm.nih.gov/geo/query/acc.cgi?acc=GSE36700 (2012).

9. Nzeusseu-Toukap, A. et al. Identification of distinct gene expression profiles in the synovium of patients with systemic lupus erythematosus. Arthritis Rheum. 56, 1579-1588 (2007).

10. Zhang, F. et al. Defining inflammatory cell states in rheumatoid arthritis joint synovial tissues by integrating single-cell transcriptomics and mass cytometry. Nat. Immunol. 20, 928-942 (2019).

11. Kuo, D. et al. HBEGF+ macrophages in rheumatoid arthritis induce fibroblast 
invasiveness. Sci. Transl. Med. 11, (2019).

12. Culemann, S. et al. Locally renewing resident synovial macrophages provide a protective barrier for the joint. Nature 572, 670-675 (2019).

13. Labonte, A. C. et al. Identification of alterations in macrophage activation associated with disease activity in systemic lupus erythematosus. PLoS One 13, e0208132 (2018).

14. Kim, E. K. et al. IL-17-mediated mitochondrial dysfunction impairs apoptosis in rheumatoid arthritis synovial fibroblasts through activation of autophagy. Cell Death Dis. 8, (2017).

15. Wang, S., Wang, L., Wu, C., Sun, S. \& Pan, J. H. E2F2 directly regulates the STAT1 and $\mathrm{PI} 3 \mathrm{~K} / \mathrm{AKT} / \mathrm{NF}-\kappa \mathrm{B}$ pathways to exacerbate the inflammatory phenotype in rheumatoid arthritis synovial fibroblasts and mouse embryonic fibroblasts. Arthritis Res. Ther. 20, (2018).

16. Mizoguchi, F. et al. Functionally distinct disease-associated fibroblast subsets in rheumatoid arthritis. Nat. Commun. 9, (2018).

17. Tellier, J. et al. Blimp-1 controls plasma cell function through the regulation of immunoglobulin secretion and the unfolded protein response. Nat. Immunol. 17, 323-30 (2016).

18. Shi, K. et al. Lymphoid chemokine B cell-attracting chemokine-1 (CXCL13) is expressed in germinal center of ectopic lymphoid follicles within the synovium of chronic arthritis patients. J. Immunol. 166, 650-5 (2001).

19. Daridon, C. et al. Splenic proliferative lymphoid nodules distinct from germinal centers are sites of autoantigen stimulation in immune thrombocytopenia. Blood 120, 5021-31 (2012). 
20. Tokunaga, R. et al. CXCL9, CXCL10, CXCL11/CXCR3 axis for immune activation - A target for novel cancer therapy. Cancer Treat. Rev. 63, 40-47 (2018).

21. Muehlinghaus, G. et al. Regulation of CXCR3 and CXCR4 expression during terminal differentiation of memory B cells into plasma cells. Blood 105, 3965-3971 (2005).

22. Buckley, C. D. et al. Persistent Induction of the Chemokine Receptor CXCR4 by TGF- $\beta 1$ on Synovial T Cells Contributes to Their Accumulation Within the Rheumatoid Synovium. J. Immunol. 165, 3423-3429 (2000).

23. Tsubaki, T. et al. Accumulation of plasma cells expressing CXCR3 in the synovial sublining regions of early rheumatoid arthritis in association with production of Mig/CXCL9 by synovial fibroblasts. Clin. Exp. Immunol. 141, 363-371 (2005).

24. Henneken, M., Dörner, T., Burmester, G. R. \& Berek, C. Differential expression of chemokine receptors on peripheral blood B cells from patients with rheumatoid arthritis and systemic lupus erythematosus. Arthritis Res. Ther. 7, 1-13 (2005).

25. de Oliveira, C. E. C. et al. CC chemokine receptor 5: the interface of host immunity and cancer. Dis. Markers 2014, 126954 (2014).

26. Zoja, C. et al. Cyclin-dependent kinase inhibition limits glomerulonephritis and extends lifespan of mice with systemic lupus. Arthritis Rheum. 56, 1629-1637 (2007).

27. Goulvestre, C. et al. A mimic of p21WAF1/CIP1 ameliorates murine lupus. J. Immunol. 175, 6959-67 (2005).

28. Dantzer, R. Neuroimmune Interactions: From the Brain to the Immune System and Vice Versa. Physiol. Rev. 98, 477-504 (2018).

29. Lahita, R. G. The immunoendocrinology of systemic lupus erythematosus. Clin. Immunol. 172, 98-100 (2016). 
30. Bereshchenko, O., Bruscoli, S. \& Riccardi, C. Glucocorticoids, Sex Hormones, and Immunity. Front. Immunol. 9, 1332 (2018).

31. Hughes, G. C. \& Choubey, D. Modulation of autoimmune rheumatic diseases by oestrogen and progesterone. Nat. Rev. Rheumatol. 10,740-751 (2014).

32. Sthoeger, Z. M., Zinger, H. \& Mozes, E. Beneficial effects of the anti-oestrogen tamoxifen on systemic lupus erythematosus of (NZBxNZW)F1 female mice are associated with specific reduction of IgG3 autoantibodies. Ann. Rheum. Dis. 62, 341-6 (2003).

33. Wu, W.-M., Lin, B.-F., Su, Y.-C., Suen, J.-L. \& Chiang, B.-L. Tamoxifen Decreases Renal Inflammation and Alleviates Disease Severity in Autoimmune NZB/W F1 Mice. Scand. J. Immunol. 52, 393-400 (2000).

34. Kim, W.-U. et al. Effect of oestrogen on T cell apoptosis in patients with systemic lupus erythematosus. Clin. Exp. Immunol. 161, $452-458$ (2010).

35. Mohammed, M. E., Nawahil, S. A., Sumaia, A. \& Abdelrahim, M. O. Tamoxifen-Induced Lupus Erythematosus. Drug Metab. Toxicol. 4, (2013).

36. Fumal, I., Danchin, A., Cosserat, F., Barbaud, A. \& Schmutz, J. L. Subacute Cutaneous Lupus erythematosus Associated with Tamoxifen Therapy: Two Cases. Dermatology 210, 251-252 (2005).

37. Chockalingam, S., Aluru, M. \& Aluru, S. Microarray Data Processing Techniques for Genome-Scale Network Inference from Large Public Repositories. Microarrays (Basel, Switzerland) 5, (2016).

38. Catalina, M. D., Bachali, P., Geraci, N. S., Grammer, A. C. \& Lipsky, P. E. Gene expression analysis delineates the potential roles of multiple interferons in systemic lupus erythematosus. Commun. Biol. 2, 140 (2019). 
39. Waddell, S. J. et al. Dissecting Interferon-Induced Transcriptional Programs in Human Peripheral Blood Cells. PLoS One 5, e9753 (2010).

40. Martinez, F. O., Gordon, S., Locati, M. \& Mantovani, A. Transcriptional Profiling of the Human Monocyte-to-Macrophage Differentiation and Polarization: New Molecules and Patterns of Gene Expression. J. Immunol. 177, 7303-7311 (2006).

41. Grammer, A. C. et al. Drug repositioning in SLE: Crowd-sourcing, literature-mining and Big Data analysis. Lupus 25, 1150-1170 (2016).

42. Hubbard, E. et al. Analysis of Lupus Synovitis Gene Expression Reveals Dysregulation of Pathogenic Pathways Activated within Infiltrating Immune Cells - ACR Meeting Abstracts. (2018). Available at: https://acrabstracts.org/abstract/analysis-of-lupussynovitis-gene-expression-reveals-dysregulation-of-pathogenic-pathways-activatedwithin-infiltrating-immune-cells/. (Accessed: 17th December 2019)

43. Hubbard, E. et al. Analysis of Gene Expression from Systemic Lupus Erythematosus Synovium Reveals Unique Pathogenic Mechanisms - ACR Meeting Abstracts. (2019). Available at: https://acrabstracts.org/abstract/analysis-of-gene-expression-from-systemiclupus-erythematosus-synovium-reveals-unique-pathogenic-mechanisms/. (Accessed: 17th December 2019)

\section{ACKNOWLEDGEMENTS}

The authors would like to thank Drs. FA Houssiau and BR Lauwerys who provided clinical data on the LA subjects studied in this report, Dr. Adam C. Labonte for his consultation on macrophage polarization and Dr. Kathryn Kingsmore Allison for her assistance with preparation of the manuscript and consultation on proper statistical technique. 
The authors would additionally like to acknowledge prior presentation of this work as posters at the ACR Annual Meetings 2018 and 2019 [42-43].

\section{AUTHOR CONTRIBUTIONS}

E. L. H. contributed to the planning and data analysis of the work described and wrote the main manuscript. M. D. C. and S. H. contributed to the planning and data analysis. P. B. and N. S. G. contributed to data analysis and software. R. R. contributed software. A. C. G. and P. E. L. contributed to the concept and design of the study, interpretation of the data, and revision and editing of the manuscript.

\section{ADDITIONAL INFORMATION}

\section{Competing Interests}

Erika Hubbard, Dr. Michelle Catalina, Sarah Heuer, Prathyusha Bachali, Robert Robl, and Dr. Nick Geraci report personal fees received for employment by AMPEL BioSolutions, LLC \& RILITE Research Institute during the preparation of this work. Drs. Amrie C. Grammer and Peter E. Lipsky report personal fees received for employment by AMPEL BioSolutions, LLC.

\section{Funding}

This work was supported by unrestricted grants from the John \& Marcia Goldman Foundation and the RILITE Research Institute. The funder provided support in the form of salaries for authors, but did not have any additional role in study design, data collection, analysis, interpretation, preparation of the manuscript, or decision to publish. 


\section{Figure 1. Overview of Gene Expression in SLE vs OA Synovium}

(a) Heatmap of 6,496 DEGs from LIMMA analysis of SLE and OA synovial gene expression data. Increased (b) and decreased (c) transcripts were each characterized by cellular signatures for prevalence of specific cell types. DE transcripts were also characterized for functional signatures. Enrichment plots in $(\mathbf{b}, \mathbf{c})$ represent odds ratios bound by $95 \%$ confidence intervals (CI) using Fisher's Exact Test. Significant enrichment by p-value $(\mathrm{p}<0.05)$ and confidence intervals that exclude odds ratio $=1$ are colored red and blue for positive or negative association with the sample, respectively. The x-axes are plotted on $\log 2$ scales. For categories represented by a single point, odds ratio $=0$ and the data point shown represents the upper bound of the confidence interval.

\section{Figure 2. IPA-Predicted Signaling Pathways and Upstream Regulators Operative in LA}

(a) Canonical pathways predicted by IPA based on DEGs, ordered by significance. (b) Significant upstream regulators predicted by IPA based on DEGs, ordered alphabetically by functional category. All canonical pathways and upstream regulators are significant by $\mid$ Activation Z-Score $\mid \geq 2$ and overlap p-value $<0.01$.

\section{Figure 3. GSVA Enrichment of Immune/Inflammatory-Specific Cells and Processes in LA}

GSVA of hematopoietic cell types (a), cytokine signatures and signaling pathways (b), immune/inflammatory processes (c), anti-inflammatory processes (d), and IPA-predicted canonical signaling pathways from DEGs in SLE vs OA synovium (e) was conducted on log2normalized gene expression values from OA and SLE synovium. Hedge's g effect sizes were 
calculated with correction for small sample size for each gene set and significant differences in enrichment between cohorts were found by Welch's t-test $(\mathrm{p}<0.05)$, shown in the panels on the right. Red and blue effect size bars represent significant enrichment in SLE and OA, respectively.

\section{Figure 4. GSVA Enrichment of Tissue-Specific Cells and Processes in LA}

GSVA of synovial tissue processes and specific cell types (a) and recently published synoviumspecific cell subtypes in human RA, OA, and mouse synovium (b-e) was conducted on log2normalized gene expression values from OA and SLE synovium. Hedge's g effect sizes were calculated with correction for small sample size for each gene set and significant differences in enrichment between cohorts were found by Welch's t-test $(\mathrm{p}<0.05)$, shown in the panels on the right. Red and blue effect size bars represent significant enrichment in SLE or OA, respectively. Literature-derived signatures in (b-e) underwent co-expression analyses before being used as GSVA gene sets (see Methods).

\section{Figure 5. Comparison of Gene Expression in SLE and RA Synovitis}

A comparison of immune/inflammatory gene signatures between SLE and RA synovium using 7 RA patients from GSE36700. (a) Upregulated DEGs were identified between RA and OA synovium, compared to DEGs from SLE vs OA synovium, and characterized by cellular signatures. GSVA of hematopoietic cell types (b), cytokine signatures and signaling pathways (c), immune/inflammatory processes (d), anti-inflammatory processes (e), and IPA-predicted canonical signaling pathways from DEGs in SLE vs OA synovium (f) was conducted on log2normalized gene expression values from SLE and RA synovium. Hedge's g effect sizes were 
calculated with correction for small sample size for each gene set and significant differences in enrichment between cohorts were found by Welch's t-test $(\mathrm{p}<0.05)$, shown in the panels on the right. Red and blue effect size bars represent significant enrichment in SLE or RA, respectively.

Figure 6. LINCS Biological Upstream Regulators and IPA Upstream Regulators Operative

\section{in LA Are Potential Druggable Targets}

(a) The top 50 targets (BURs) opposing the LA gene signature from LINCS knock down (KD) and overexpression (OE) assays summarized by connectivity score and matched to appropriate targeting drugs. $\mathrm{KD}$ and $\mathrm{OE}$ data were filtered for connectivity scores in the $[-75$ to -100$]$ and $[50$ to 100] ranges, respectively. (b) The consensus IPA-predicted UPRs between DEGs and LAassociated WGCNA modules summarized by Activation Z-Score, functional category, and also matched to appropriate targeting drugs. Drugs and compounds targeting the BURs and UPRs were sourced from LINCS/CLUE, IPA, literature mining, CoLTS [41], STITCH, and clinical trials databases. Drug annotations are grouped together by target and CoLTS scores (range -16 to +11 ) are displayed as integers in superscript. Some upstream regulators are matched to groups of drugs (e.g., NFkB pathway inhibitors, bold, italicized), for which the full list of drug-target matches can be found in Supplementary Data S15-16 online. ${ }^{\mathbf{P}}$ : Preclinical; ${ }^{\ddagger}$ : Drug in development/clinical trials; ${ }^{\dagger}$ : FDA-approved 
bioRxiv preprint doi: https://doi.org/10.1101/2020.06.19.123307; this version posted June 21, 2020. The copyright holder for this preprint (which was not certified by peer review) is the author/funder. All rights reserved. No reuse allowed without permission.

Table 1. Chemokine Receptor-Ligand Pairs and Adhesion Molecules Associated with LA

\begin{tabular}{|c|c|c|c|}
\hline \multirow[b]{2}{*}{$\begin{array}{c}\text { Gene } \\
\text { Transcript }\end{array}$} & \multirow[b]{2}{*}{ Name } & \multicolumn{2}{|c|}{ SLE vs OA Analysis } \\
\hline & & DE LFC & $\begin{array}{c}\text { WGCNA } \\
\text { LA-associated module }\end{array}$ \\
\hline CCL19 & Chemokine (C-C motif) ligand 19 & $\mathrm{n} / \mathrm{s}$ & Honeydew 1 \\
\hline CCR2 & Chemokine (C-C motif) receptor 2 & 1.50 & Midnightblue \\
\hline CCL2 & Chemokine (C-C motif) ligand 2 & $\mathrm{n} / \mathrm{s}$ & Darkgrey \\
\hline CCL7 & Chemokine (C-C motif) ligand 7 & $\mathrm{n} / \mathrm{s}$ & Darkgrey \\
\hline CCL8 & Chemokine (C-C motif) ligand 8 & 2.76 & Darkgrey \\
\hline CCR5 & Chemokine (C-C motif) receptor 5 & 1.90 & Navajowhite2 \\
\hline CCL4 & Chemokine (C-C motif) ligand 4 & 2.67 & Brown \\
\hline CCL5 & Chemokine (C-C motif) ligand 5 & 1.94 & Midnightblue \\
\hline CCL8 & Chemokine (C-C motif) ligand 8 & 2.76 & Darkgrey \\
\hline CCR1 & Chemokine (C-C motif) receptor 1 & 2.14 & Brown \\
\hline CCL5 & Chemokine (C-C motif) ligand 5 & 1.94 & Midnightblue \\
\hline CCL7 & Chemokine (C-C motif) ligand 7 & $\mathrm{n} / \mathrm{s}$ & Darkgrey \\
\hline CCL8 & Chemokine (C-C motif) ligand 8 & 2.76 & Darkgrey \\
\hline CCL23 & Chemokine (C-C motif) ligand 23 & 0.670 & \\
\hline CCR3 & Chemokine (C-C motif) receptor 3 & $\mathrm{n} / \mathrm{s}$ & Darkgrey \\
\hline CCL5 & Chemokine (C-C motif) ligand 5 & 1.94 & Midnightblue \\
\hline CCL7 & Chemokine (C-C motif) ligand 7 & $\mathrm{n} / \mathrm{s}$ & Darkgrey \\
\hline CCL8 & Chemokine (C-C motif) ligand 8 & 2.76 & Darkgrey \\
\hline CCRL2 & Chemokine (C-C motif) receptor-like 2 & 1.04 & Navajowhite2 \\
\hline CKLF & Chemokine like factor & 0.297 & \\
\hline CMKLR1 & Chemokine-like receptor 1 & 1.59 & Darkgrey, Honeydew1 \\
\hline CXCL2 & Chemokine (C-X-C motif) ligand 2 & 2.98 & Honeydew1 \\
\hline CXCL3 & Chemokine (C-X-C motif) ligand 3 & 1.77 & \\
\hline CXCL8 & Chemokine (C-X-C motif) ligand 8 & 2.17 & Brown, Darkgrey \\
\hline CXCR3 & Chemokine (C-X-C motif) receptor 3 & 1.45 & Midnightblue \\
\hline CXCL9 & Chemokine (C-X-C motif) ligand 9 & 5.59 & Midnightblue \\
\hline CXCL10 & Chemokine (C-X-C motif) ligand 10 & 4.81 & Midnightblue \\
\hline CXCL11 & Chemokine (C-X-C motif) ligand 11 & 3.32 & Midnightblue \\
\hline CXCR4 & Chemokine (C-X-C motif) receptor 4 & 1.39 & Brown \\
\hline CXCL13 & Chemokine (C-X-C motif) ligand 13 & 3.47 & Midnightblue \\
\hline CXCR6 & Chemokine (C-X-C motif) receptor 6 & $\mathrm{n} / \mathrm{s}$ & Midnightblue \\
\hline CXCL16 & Chemokine (C-X-C motif) ligand 16 & 0.768 & Navajowhite2 \\
\hline CXCL11 & Chemokine (C-X-C motif) ligand 11 & 3.32 & Midnightblue \\
\hline CX3CL1 & Chemokine (C-X3-C motif) ligand 1 & 0.453 & \\
\hline XCL1 & Chemokine (X-C motif) ligand 1 & $\mathrm{n} / \mathrm{s}$ & Midnightblue \\
\hline ALCAM & Activated leukocyte cell adhesion molecule & 1.55 & \\
\hline VCAM1 & Vascular cell adhesion molecule 1 & $\mathrm{n} / \mathrm{s}$ & Navajowhite2 \\
\hline CD44 & CD44 molecule & 1.25 & Brown, Darkgrey \\
\hline ITGB1 & Integrin subunit beta 1 & -0.255 & \\
\hline ITGB2 & Integrin subunit beta 2 & 1.56 & Brown, Honeydew1 \\
\hline ICAM1 & Intercellular adhesion molecule 1 & 0.861 & $\begin{array}{c}\text { Darkgrey, Honeydew1, } \\
\text { Midnightblue }\end{array}$ \\
\hline ICAM3 & Intercellular adhesion molecule 3 & $\mathrm{n} / \mathrm{s}$ & Midnightblue \\
\hline PECAM1 & Platelet/endothelial cell adhesion molecule 1 & 0.618 & Salmon4 \\
\hline SDK1 & Sidekick cell adhesion molecule 1 & -0.892 & \\
\hline SDK2 & Sidekick cell adhesion molecule 2 & -1.33 & \\
\hline CADM1 & Cell adhesion molecule 1 & -0.974 & \\
\hline CADM3 & Cell adhesion molecule 3 & $\mathrm{n} / \mathrm{s}$ & Darkgrey \\
\hline JAM2 & Junctional adhesion molecule 2 & -0.587 & \\
\hline JAM3 & Junctional adhesion molecule 3 & -0.673 & \\
\hline MCAM & Melanoma cell adhesion molecule & -1.08 & \\
\hline
\end{tabular}

DEGs and LA-associated WGCNA modules were assessed for adhesion molecules and

chemokine receptor-ligand pairs. Receptor-ligand pairs are grouped together in the table with 
bioRxiv preprint doi: https://doi.org/10.1101/2020.06.19.123307; this version posted June 21, 2020. The copyright holder for this preprint (which was not certified by peer review) is the author/funder. All rights reserved. No reuse allowed without permission.

groupings alternately shaded. Log fold changes rounded to 3 significant figures are presented where available; otherwise, $\mathrm{n} / \mathrm{s}=$ not significant. 
bioRxiv preprint doi: https://doi.org/10.1101/2020.06.19.123307; this version posted June 21, 2020. The copyright holder for this preprint (which was not certified by peer review) is the author/funder. All rights reserved. No reuse allowed without permission.

\section{Table 2. Compounds Targeting LA}

\begin{tabular}{|c|c|c|c|c|c|}
\hline Target & Count & $\underline{\text { Range }}$ & $\underline{\text { Mean } \pm \text { SEM }}$ & Top LINCS Drug & $\frac{\text { Representative }}{\text { Drug }}$ \\
\hline PKC & 5 & $(-94.47)-(-99.70)$ & $-98.01 \pm 1.00$ & Enzastaurin $^{*}$ & Midostaurin $^{\dagger 0}$ \\
\hline STK33 & 2 & $(-94.85)-(-95.45)$ & $-95.15 \pm 0.30$ & MW-STK33-1A & ML-281 ${ }^{\mathrm{P}}$ \\
\hline RAF & 3 & $(-89.13)-(-98.35)$ & $-94.91 \pm 2.91$ & Vemurafenib $^{\dagger-6}$ & Sorafenib $^{\dagger-3}$ \\
\hline GSK3 & 7 & $(-81.19)-(-99.96)$ & $-93.93 \pm 2.57$ & SB-216763 ${ }^{\mathrm{P}}$ & Enzastaurin ${ }^{*}$ \\
\hline ROCK $1 / 2$ & 4 & $(-90.80)-(-97.82)$ & $-92.81 \pm 1.68$ & RHO-kinase-inhibitor-III & $\mathrm{KD}^{2} 25^{\dagger 7}$ \\
\hline NOS2 & 3 & $(-83.31)-(-98.09)$ & $-92.46 \pm 4.61$ & AR-C133057XX & Curcumin $^{\dagger 7}$ \\
\hline CDK & 7 & $(-81.19)-(-99.96)$ & $-91.95 \pm 2.43$ & SB-216763 ${ }^{P}$ & Palbociclib $^{\dagger 4}$ \\
\hline GR agonist & 11 & $(-83.48)-(-97.95)$ & $-91.61 \pm 1.53$ & Dexamethasone $^{\dagger}$ & Prednisone $^{\dagger}$ \\
\hline PLK & 2 & $(-84.06)-(-99.04)$ & $-91.55 \pm 7.49$ & GW-843682X $\mathrm{X}^{\mathbf{P}}$ & Rigosertib \\
\hline $\begin{array}{l}\text { Dopamine uptake } \\
\text { (SLC6A3) }\end{array}$ & 2 & $(-86.38)-(-95.84)$ & $-91.11 \pm 4.73$ & GBR-13069 & Bupropion ${ }^{\dagger}$ \\
\hline Cholinesterase & 2 & $(-88.16)-(-93.36)$ & $-90.76 \pm 2.60$ & Mestinon $^{\dagger}$ & Isoflurophate ${ }^{\dagger}$ \\
\hline Retinoid $\mathrm{R}$ agonist & 5 & $(-81.80)-(-95.44)$ & $-90.34 \pm 2.40$ & TTNPB & Acitretin ${ }^{\dagger}$ \\
\hline Tyrosine kinase & 4 & $(-86.44)-(-97.25)$ & $-90.24 \pm 2.42$ & Sorafenib $^{\dagger-3}$ & Nilotinib $^{\dagger 0}$ \\
\hline $\mathrm{mTORC} 1 / 2$ & 4 & $(-88.13)-(-91.96)$ & $-90.10 \pm 0.87$ & KU-0063794 ${ }^{\mathrm{P}}$ & $\mathrm{N}$-acetyl cysteine ${ }^{i 4}$ \\
\hline Tubulin & 19 & $(-82.65)-(-96.57)$ & $-89.67 \pm 1.10$ & Epothilone & Albendazole $^{\dagger}$ \\
\hline Aurora kinase & 3 & $(-81.99)-(-96.22)$ & $-89.59 \pm 4.14$ & AT-9283 & Alisertib \\
\hline SYK & 2 & $(-85.66)-(-93.43)$ & $-89.55 \pm 3.88$ & Fostamatinib $^{\lceil 7}$ & Fostamatinib $^{\dagger 7}$ \\
\hline EGFR & 12 & $(-79.42)-(-99.14)$ & $-89.48 \pm 1.85$ & Lapatinib $^{\dagger 0}$ & Gefitinib $^{\dagger 1}$ \\
\hline $\begin{array}{l}\text { b2 adrenergic } \mathrm{R} \\
\text { agonist }\end{array}$ & 5 & $(-82.19)-(-96.66)$ & $-88.82 \pm 2.58$ & Orciprenaline & Albuterol $^{\dagger}$ \\
\hline 5 alpha reductase & 2 & $(-86.29)-(-91.18)$ & $-88.73 \pm 2.44$ & Alpha-estradiol & Acexamic acid $^{\dagger}$ \\
\hline c-Met & 2 & $(-81.96)-(-94.78)$ & $-88.37 \pm 6.41$ & SU-11274 & Cabozantinib $^{\dagger-6}$ \\
\hline PARP & 6 & $(-77.24)-(-98.35)$ & $-88.28 \pm 3.17$ & Rucaparib $^{\ddagger}$ & Niraparib $^{\dagger 3}$ \\
\hline Angiotensin R & 2 & $(-84.35)-(-92.20)$ & $-88.28 \pm 3.93$ & Candesartan $^{\dagger}$ & Azilsartan $^{\dagger}$ \\
\hline MDM & 5 & $(-75.96)-(-95.49)$ & $-88.13 \pm 3.48$ & SJ-172550 ${ }^{\mathbf{P}}$ & Idasanutlin \\
\hline MAO & 2 & $(-83.30)-(-92.34)$ & $-87.82 \pm 4.52$ & Nialamide $^{\wedge}$ & Bifemelane $^{\dagger}$ \\
\hline $\begin{array}{l}\mathrm{Na} / \mathrm{Ca} \text { exchange } \\
\quad(\mathrm{SLC} 8 \mathrm{~A} 1)\end{array}$ & 2 & $(-86.48)-(-88.99)$ & $-87.73 \pm 1.26$ & CGP-37157 ${ }^{\mathrm{P}}$ & CGP-37157 ${ }^{\mathrm{P}}$ \\
\hline VEGFR & 4 & $(-77.30)-(-97.26)$ & $-87.70 \pm 4.52$ & Sorafenib $^{\dagger-3}$ & Sunitinib $^{\dagger 1}$ \\
\hline Na channel & 11 & $(-79.66)-(-98.23)$ & $-87.62 \pm 1.64$ & Phenamil & Benzocaine $^{\dagger}$ \\
\hline TRPV agonist & 3 & $(-80.20)-(-97.26)$ & $-87.56 \pm 5.06$ & Capsaicin $^{\dagger}$ & Capsaicin $^{\dagger}$ \\
\hline P450 & 3 & $(-81.70)-(-92.02)$ & $-87.53 \pm 3.06$ & Proadifen $^{\mathbf{P}}$ & Resveratrol $^{45}$ \\
\hline MAP2K1/2 & 10 & $(-80.30)-(-98.40)$ & $-87.44 \pm 1.93$ & PD-0325901 & Vemurafenib $^{\dagger-6}$ \\
\hline Androgen R & 5 & $(-81.04)-(-96.04)$ & $-87.36 \pm 2.70$ & BMS-641988 & Apalutamide $^{\dagger}$ \\
\hline Adenosine $\mathrm{R}$ & 2 & $(-79.87)-(-94.23)$ & $-87.05 \pm 7.18$ & ZM-241385 ${ }^{\text {P }}$ & Caffeine $^{\dagger}$ \\
\hline HIV protease & 2 & $(-86.19)-(-87.78)$ & $-86.98 \pm 0.79$ & Lopinavir & Nelfinavir $^{\dagger 5}$ \\
\hline HMG-CoA reductase & 5 & $(-76.32)-(-93.09)$ & $-86.97 \pm 2.85$ & Atorvastatin $^{\dagger 3}$ & Statins $^{\dagger 3}$ \\
\hline TGFBR & 3 & $(-80.73)-(-98.13)$ & $-86.93 \pm 5.61$ & SB-525334 & Pirfenidone $^{\dagger}$ \\
\hline Tachykinin & 3 & $(-80.84)-(-91.26)$ & $-86.39 \pm 3.03$ & Aprepitant $^{\dagger}$ & Aprepitant $^{\dagger}$ \\
\hline PRKDC & 3 & $(-83.42)-(-90.00)$ & $-86.04 \pm 2.02$ & NU-7026 & Caffeine $^{\dagger}$ \\
\hline NSAID/prostaglandin & 13 & $(-76.19)-(-95.40)$ & $-86.04 \pm 1.58$ & Phenacetin ${ }^{\wedge}$ & Aspirin ${ }^{\dagger}$ \\
\hline MAPK & 10 & $(-77.41)-(-95.39)$ & $-85.42 \pm 1.89$ & FR-180204 ${ }^{\mathrm{P}}$ & Losmapimod $^{*}$ \\
\hline TP53 & 2 & $(-78.14)-(-92.39)$ & $-85.27 \pm 7.13$ & Pifithrin-alpha $^{\mathbf{P}}$ & VLX-600" \\
\hline Prostanoid $\mathrm{R}$ agonist & 2 & $(-82.17)-(-87.80)$ & $-84.99 \pm 2.82$ & $\begin{array}{l}\text { 16,16-dimethylprostaglandin- } \\
\mathrm{e} 2^{\ddagger}\end{array}$ & Alprostadil ${ }^{\dagger}$ \\
\hline PDE & 9 & $(-77.14)-(-95.12)$ & $-84.73 \pm 2.42$ & Bucladesine $^{\dagger}$ & Dipyridamole $^{\dagger 4}$ \\
\hline ATM kinase & 2 & $(-83.89)-(-85.10)$ & $-84.50 \pm 0.61$ & $\mathrm{CP} 466722$ & VE- $822^{*}$ \\
\hline PI3K (pan) & 3 & $(-78.72)-(-88.90)$ & $-84.11 \pm 2.95$ & PIK-90 & Idelalisib $^{\not 1}$ \\
\hline ACE & 2 & $(-81.90)-(-86.18)$ & $-84.04 \pm 2.14$ & Enalapril $^{\dagger}$ & Alacepril $^{\dagger}$ \\
\hline DHFR & 2 & $(-75.51)-(-92.42)$ & $-83.96 \pm 8.45$ & Pyrimethamine $^{\dagger}$ & Methotrexate $^{\dagger}$ \\
\hline NFkB & 3 & $(-76.88)-(-89.33)$ & $-83.94 \pm 3.69$ & NFKB-activation-inhibitor-II ${ }^{\mathbf{P}}$ & $\mathrm{N}$-acetyl cysteine $\mathrm{i}^{\dagger 4}$ \\
\hline ALOX5 & 2 & $(-76.07)-(-89.98)$ & $-83.03 \pm 6.95$ & Zilueton $^{\dagger}$ & Diethylcarbamazine $^{\dagger}$ \\
\hline DNMT & 3 & $(-75.19)-(-88.82)$ & $-82.48 \pm 3.96$ & Triclosan $^{\dagger}$ & Azacitidine $^{\dagger}$ \\
\hline HDAC & 4 & $(-77.36)-(-90.05)$ & $-82.32 \pm 2.75$ & Valproic acid $^{\dagger 2}$ & Vorinostat $^{\dagger 6}$ \\
\hline AMPA R agonist & 2 & $(-79.16)-(-84.63)$ & $-81.90 \pm 2.74$ & Nobiletin $^{\mathbf{P}}$ & Aniracetam $^{\dagger}$ \\
\hline Topoisomerase II & 3 & $(-77.89)-(-88.85)$ & $-81.87 \pm 3.50$ & Razoxane & Doxorubicin $^{\dagger}$ \\
\hline IGF1R & 2 & $(-78.32)-(-83.82)$ & $-81.07 \pm 2.75$ & GSK-1904529A $A^{P}$ & Ceritinib $^{\dagger-4}$ \\
\hline HSP90AA1 & 2 & $(-76.84)-(-85.28)$ & $-81.06 \pm 4.22$ & Gedunin $^{\mathbf{P}}$ & Rifabutin $^{\dagger}$ \\
\hline NAMPT & 2 & $(-76.91)-(-84.58)$ & $-80.75 \pm 3.83$ & FK-866 & FK-866 \\
\hline Calcineurin & 2 & $(-79.03)-(-80.44)$ & $-79.73 \pm 0.70$ & 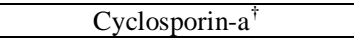 & Tacrolimus $^{+5}$ \\
\hline Carbonic anhydrase & 2 & $(-78.54)-(-78.81)$ & $-78.67 \pm 0.13$ & Chlortalidone $^{\dagger}$ & Acetazolamide $^{\dagger}$ \\
\hline
\end{tabular}


Compounds predicted by LINCS to oppose the LA gene signature were summarized by their drug targets for every target with at least two compounds. Compounds were analyzed if corresponding connectivity scores fell in the range of -75 to -100 to reflect most opposite gene signatures. Top LINCS Drug represents the most negative-scoring compound for a specific target category. Representative Drug represents the most immunologically relevant or well-known drug for a specific target category. Where applicable, CoLTS scores (range -16 to +11 ) [41] are displayed as integers in superscript. $\mathrm{R}=$ receptor; ${ }^{\mathbf{P}}$ : Preclinical; ${ }^{\ddagger}$ : Drug in development/clinical trials; ${ }^{\dagger}$ : FDA-approved; ’ : Withdrawn from market 
a

\begin{tabular}{c|c} 
SLE & $\begin{array}{l}\text { SLE_9021 } \\
\text { SLE_9022 } \\
\text { SLE_9023 } \\
\text { SLE_9024 }\end{array}$ \\
OA & $\begin{array}{l}\text { OA_8470 } \\
\text { OA_9012 } \\
\text { OA_9011 } \\
\text { OA_9010 }\end{array}$
\end{tabular}

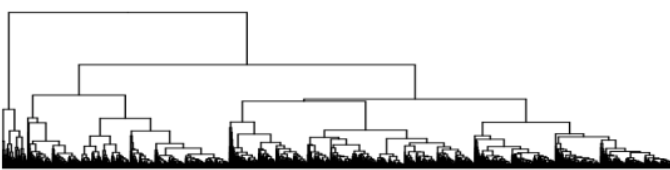

b

SLE vs OA Up DEGs
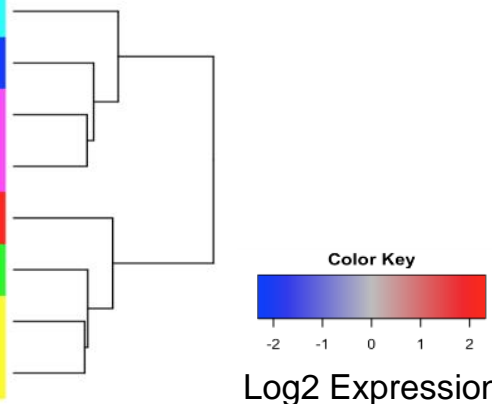

Log2 Expression
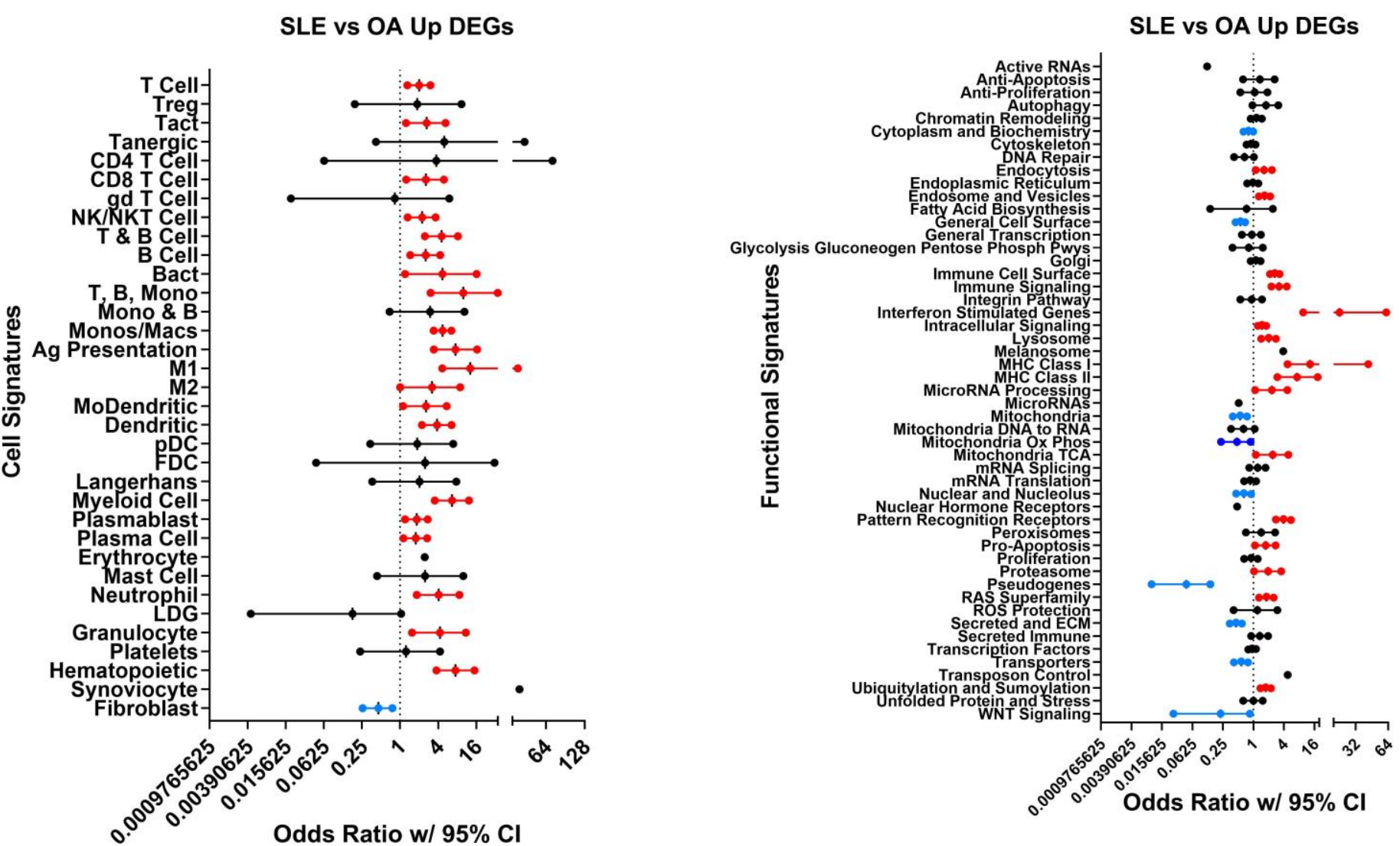

C

SLE vs OA Down DEGs

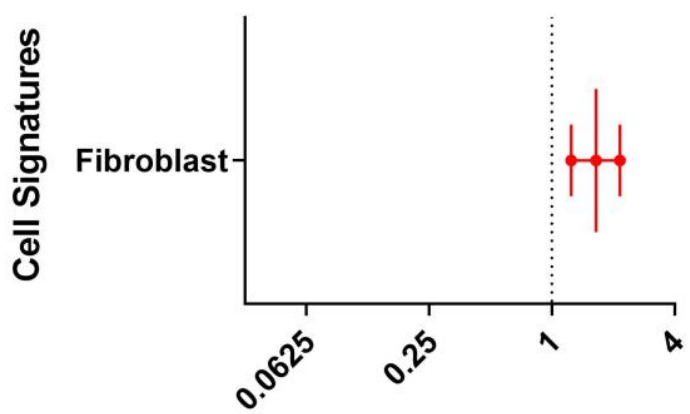

SLE vs OA Down DEGs

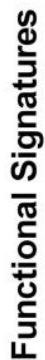

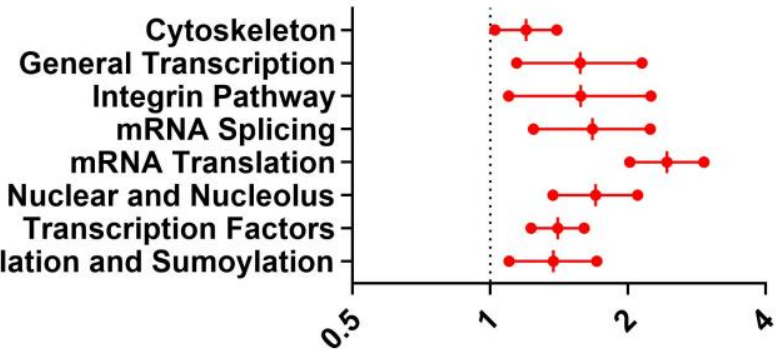

Odds Ratio w/ $95 \% \mathrm{Cl}$

Odds Ratio w/ 95\% Cl 


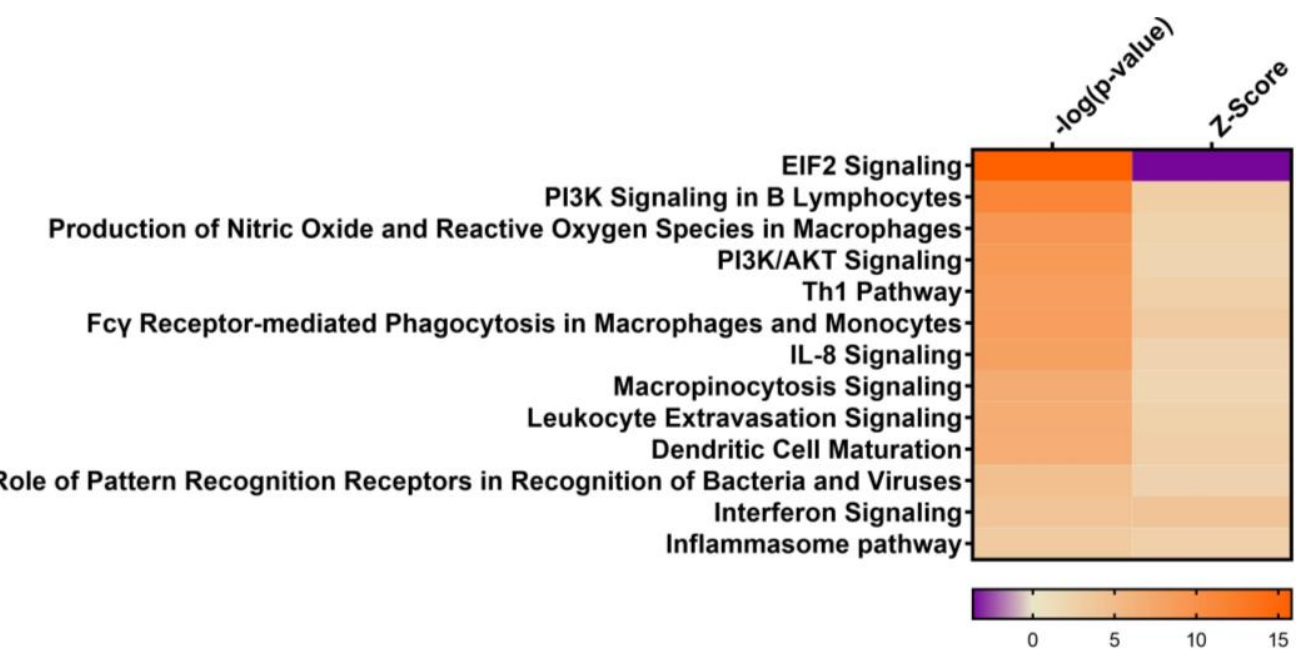

b IPA Upstream Regulators

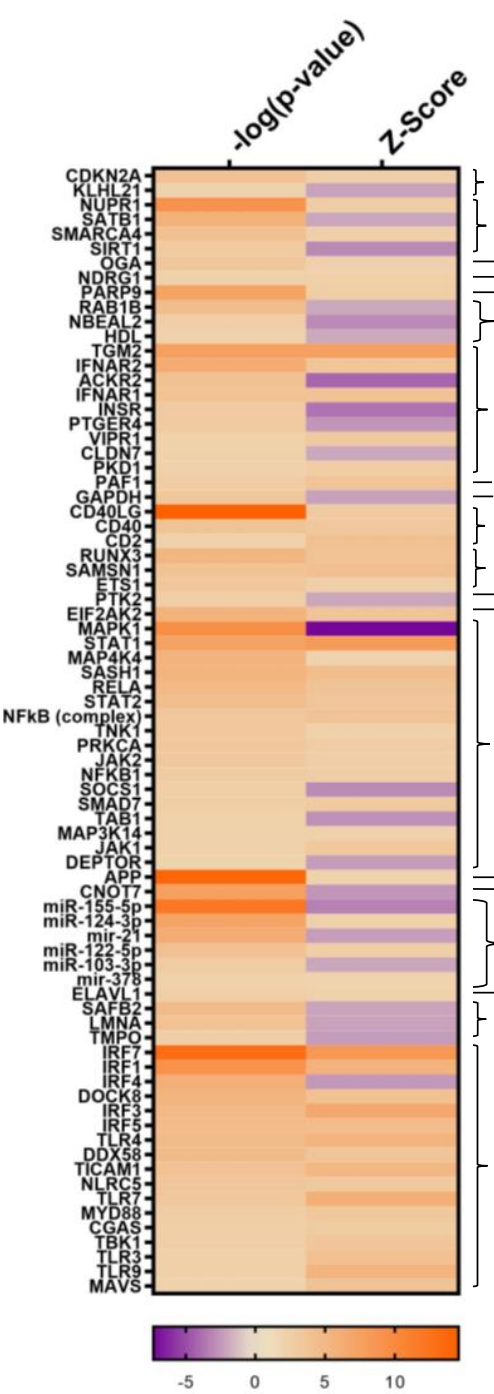

Functional Signature

Anti-Proliferation

- Chromatin Remodeling

Cytoplasm \& Biochemistry

ER

General Cell Surface

- General Transcription

Glycol., Gluconeogen. \& Pent. Phosph. Pwy:

Immune Cell Surface

Immune Signaling

= Integrin Pathway

Intracellular Signaling

ㄴysosome

- MicroRNAs

mRNA Translation

Nucleus and Nucleolus

Pattern Recognition Receptors

\section{IPA Upstream Regulators

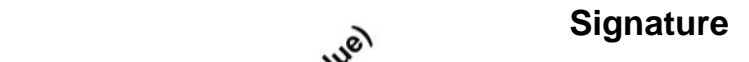


a Hematopoietic Cell Types
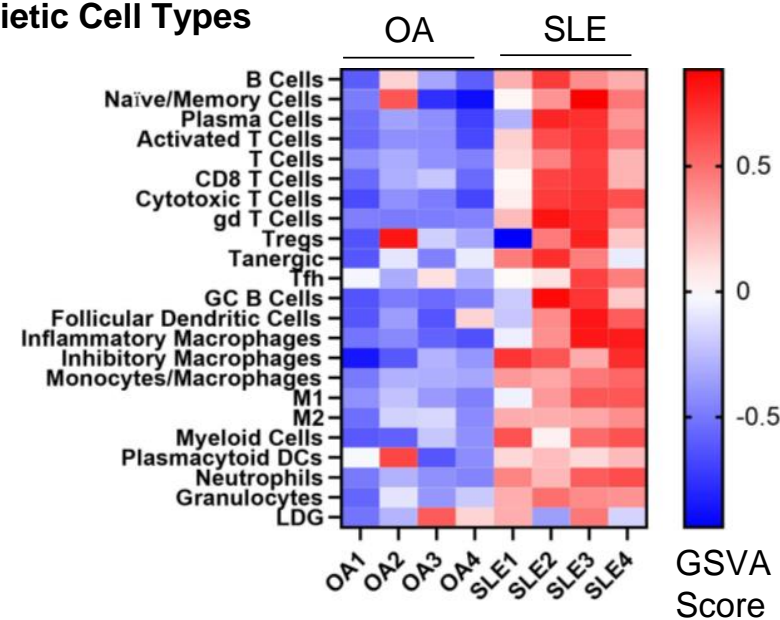

\section{b Cytokines/Cytokine Signaling}

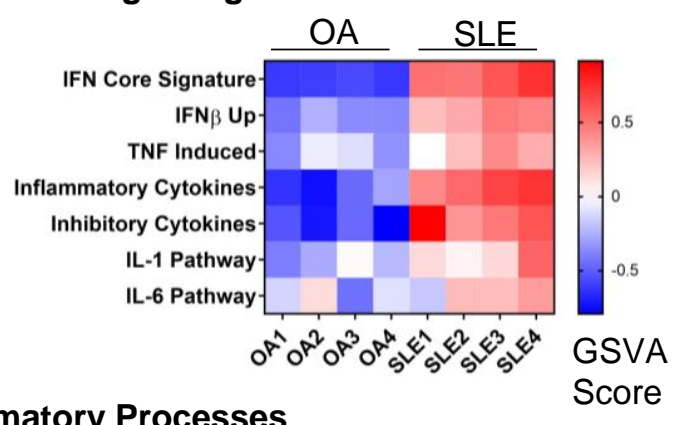

\section{c Immune/Inflammatory Processes}

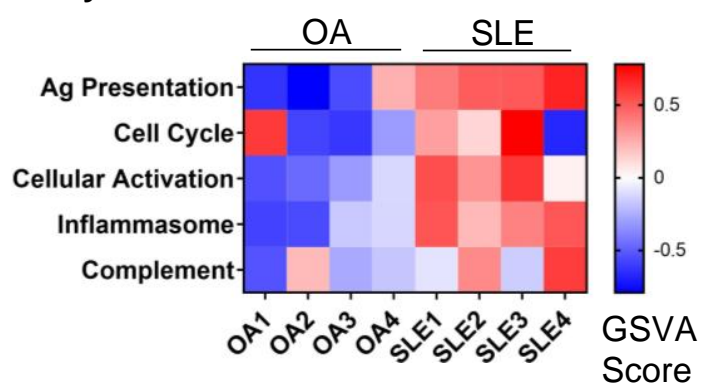

\section{d Anti-inflammation}

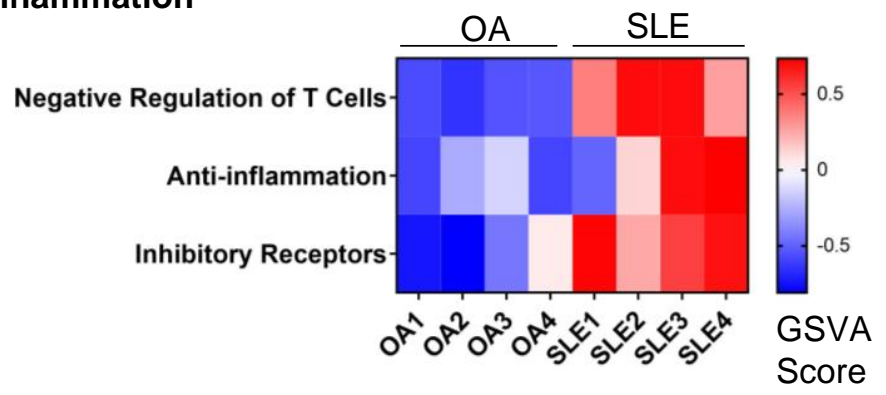

\section{e IPA Canonical Pathways}

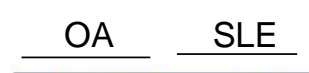

PI3K Signaling SignalingPI3K Signaling in B Lymphocytes-
Production of NO and ROS in MacrophagesPI3KIAKT Signaling

Fc $\gamma$ R-mediated Phagocytosis in Monos/Macs IL-8 Signaling-

Macropinocytosis Signaling Leukocyte Extravasation SignalingDendritic Cell MaturationInterferon SignalingInflammasome PathwayB Cell Receptor Signaling-

fMLP Signaling in NeutrophilsSignaling by Rho Family GTPasesCXCR4 SignalingJak/Stat Signaling Tec Kinase Signaling

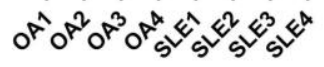
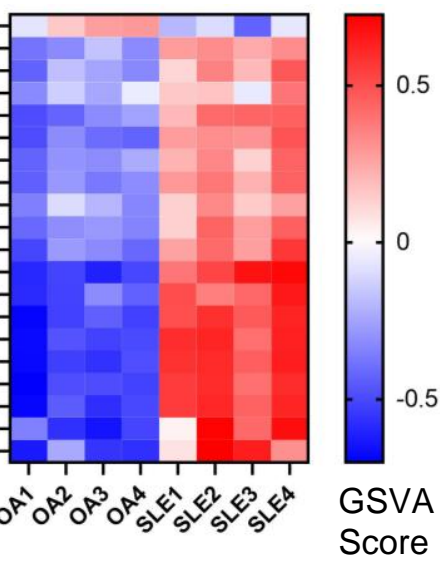

Follicular Dendritic Cells Inflammatory MacrophagesInhibitory Macrophages Monocytes/Macrophages
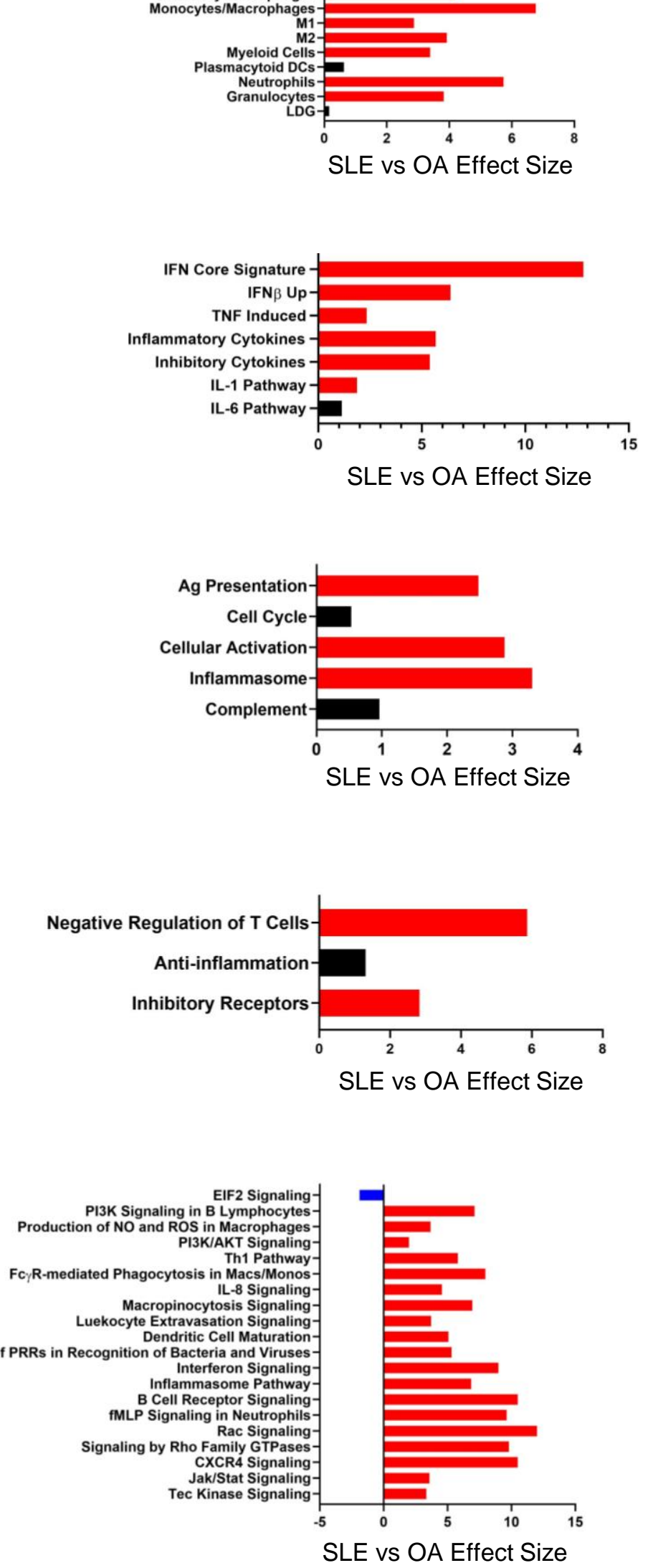
a Tissue
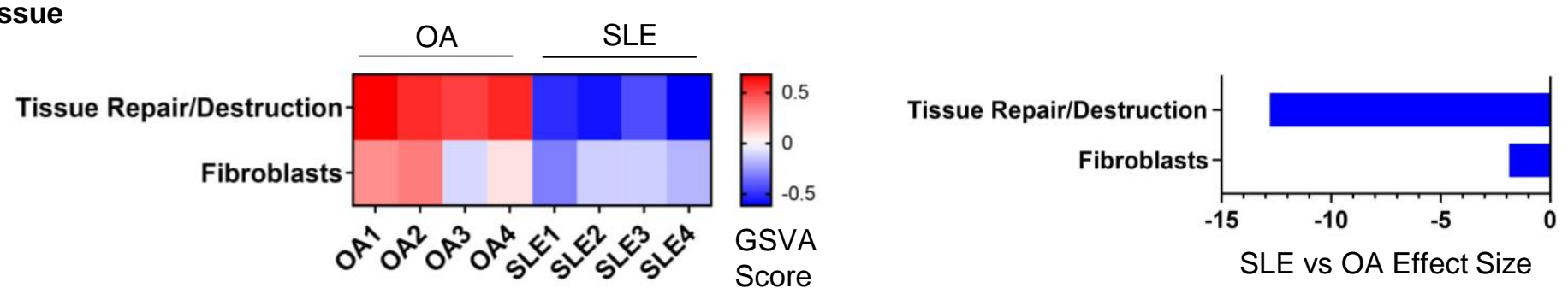

b Single Cell Fibroblast Populations
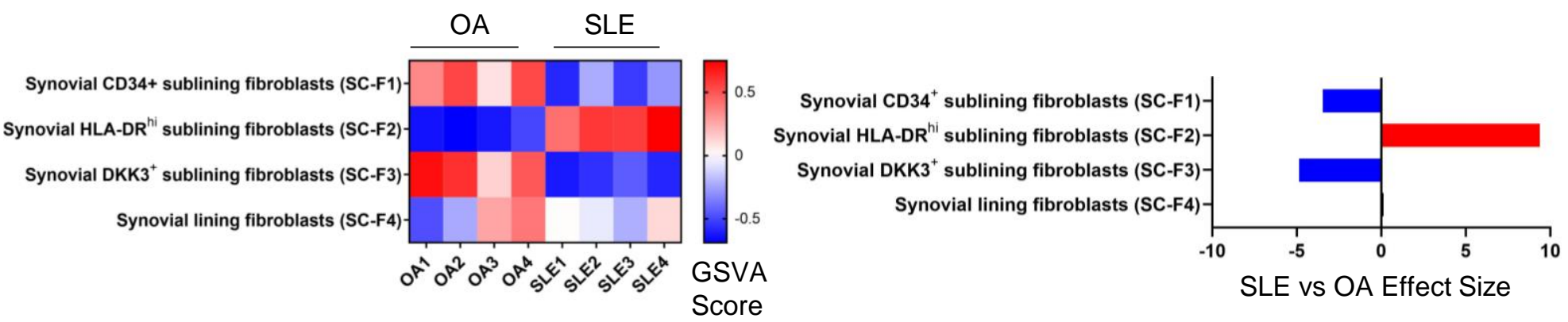

\section{c Single Cell Macrophage Populations}

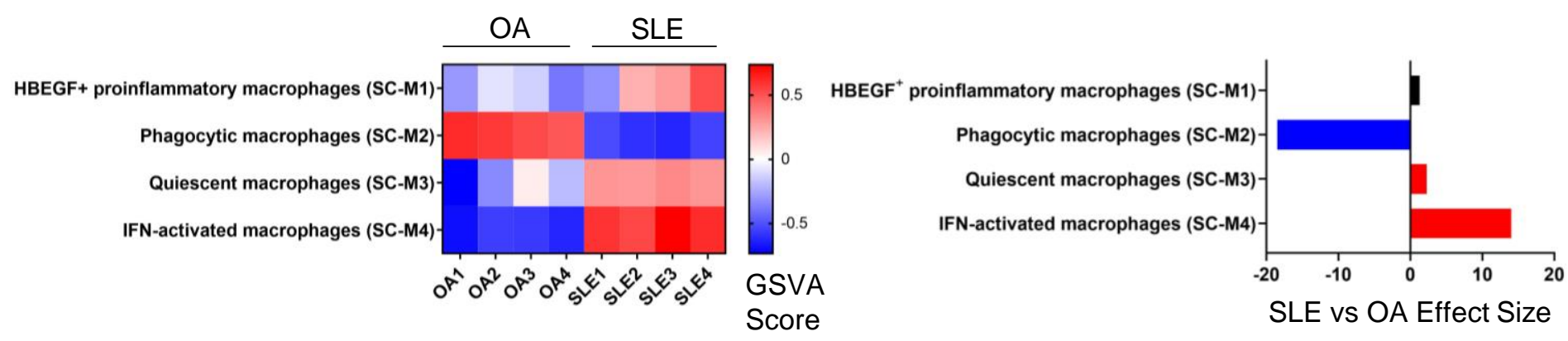

\section{d Control Mouse Synovial Cell Populations}
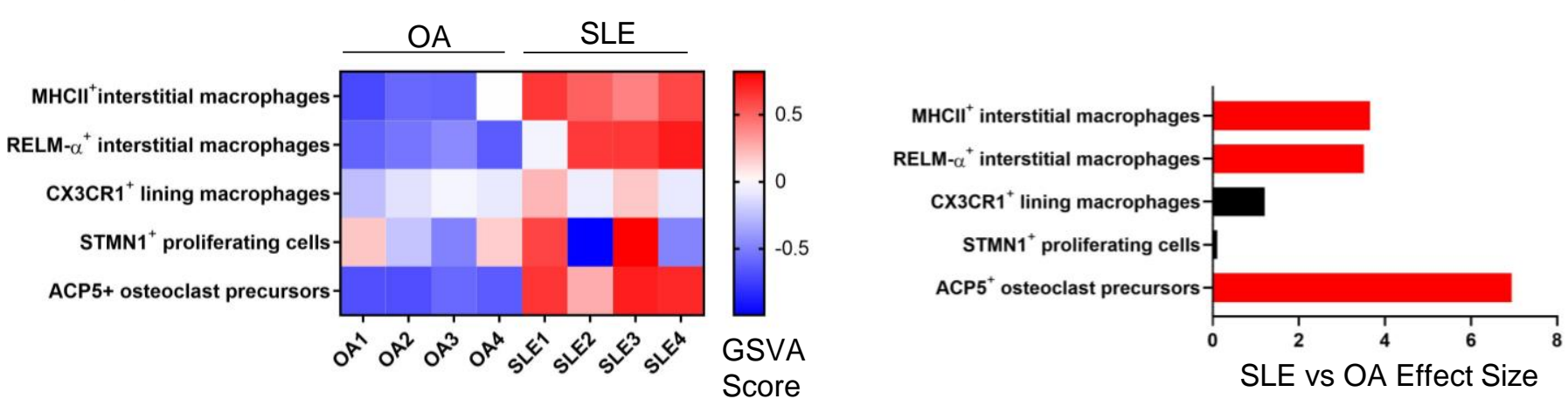

\section{e Mouse Synovial Cell Populations after Serum Transfer Arthritis Induction}
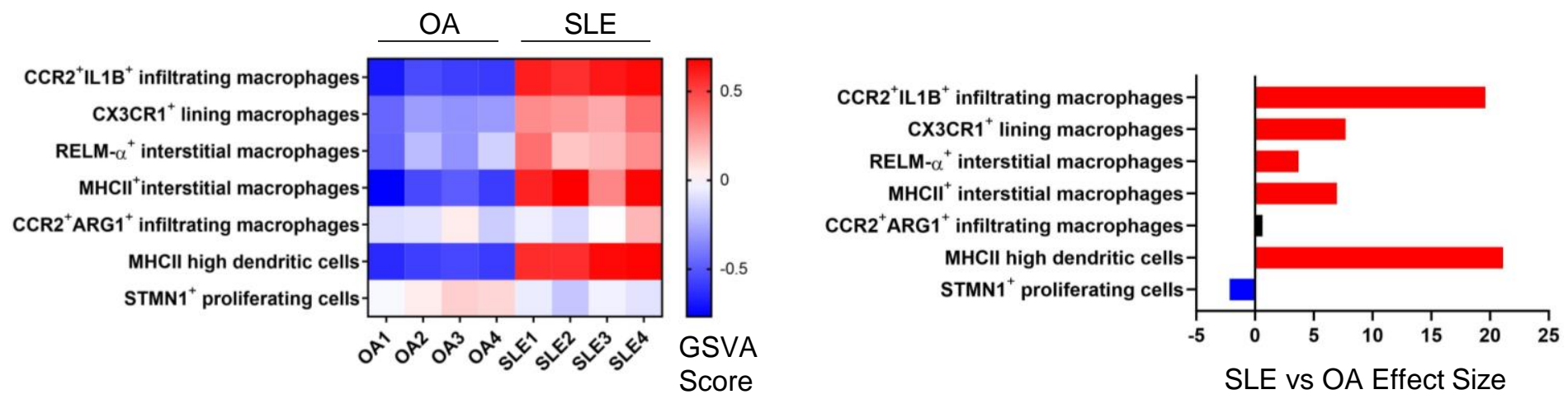
a Comparison of Synovial Immune Infiltrates
Up DEGs

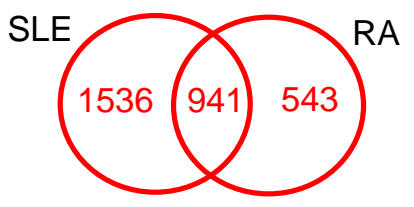

b Hematopoietic Cell Types

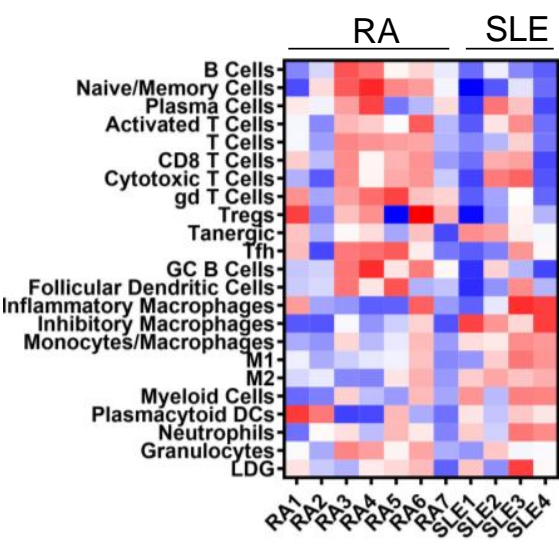

Up Immune/Inflammatory Cell-Specific DEGs

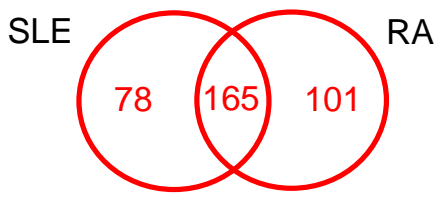

c Cytokines/Cytokine Signaling
IFN Core Signature-

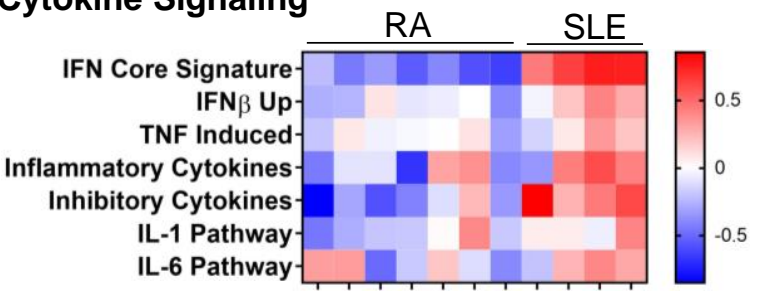

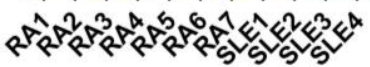

d Immune/Inflammatory Processes

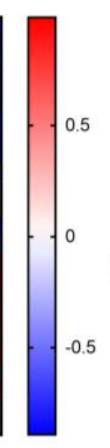

GSVA

Score

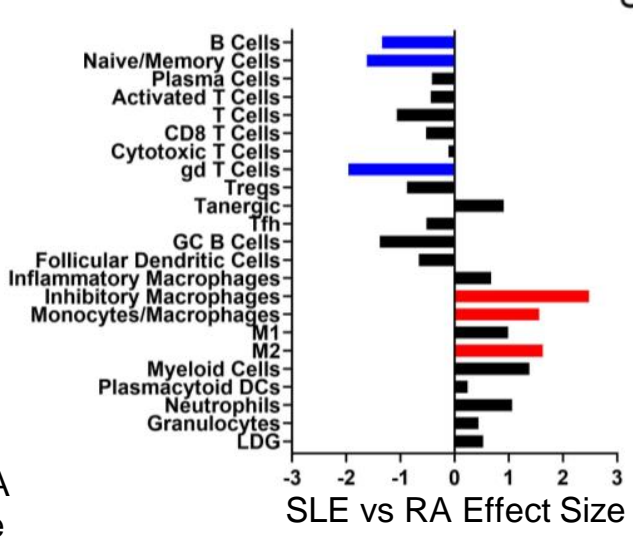

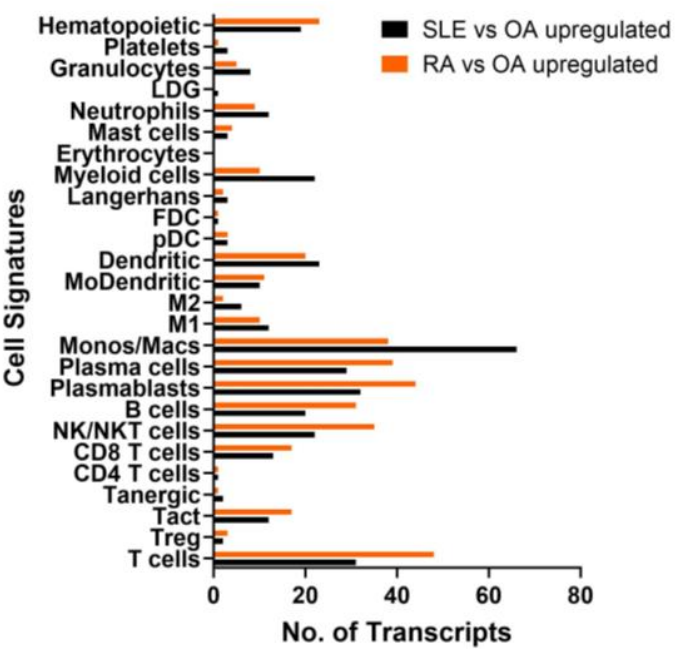
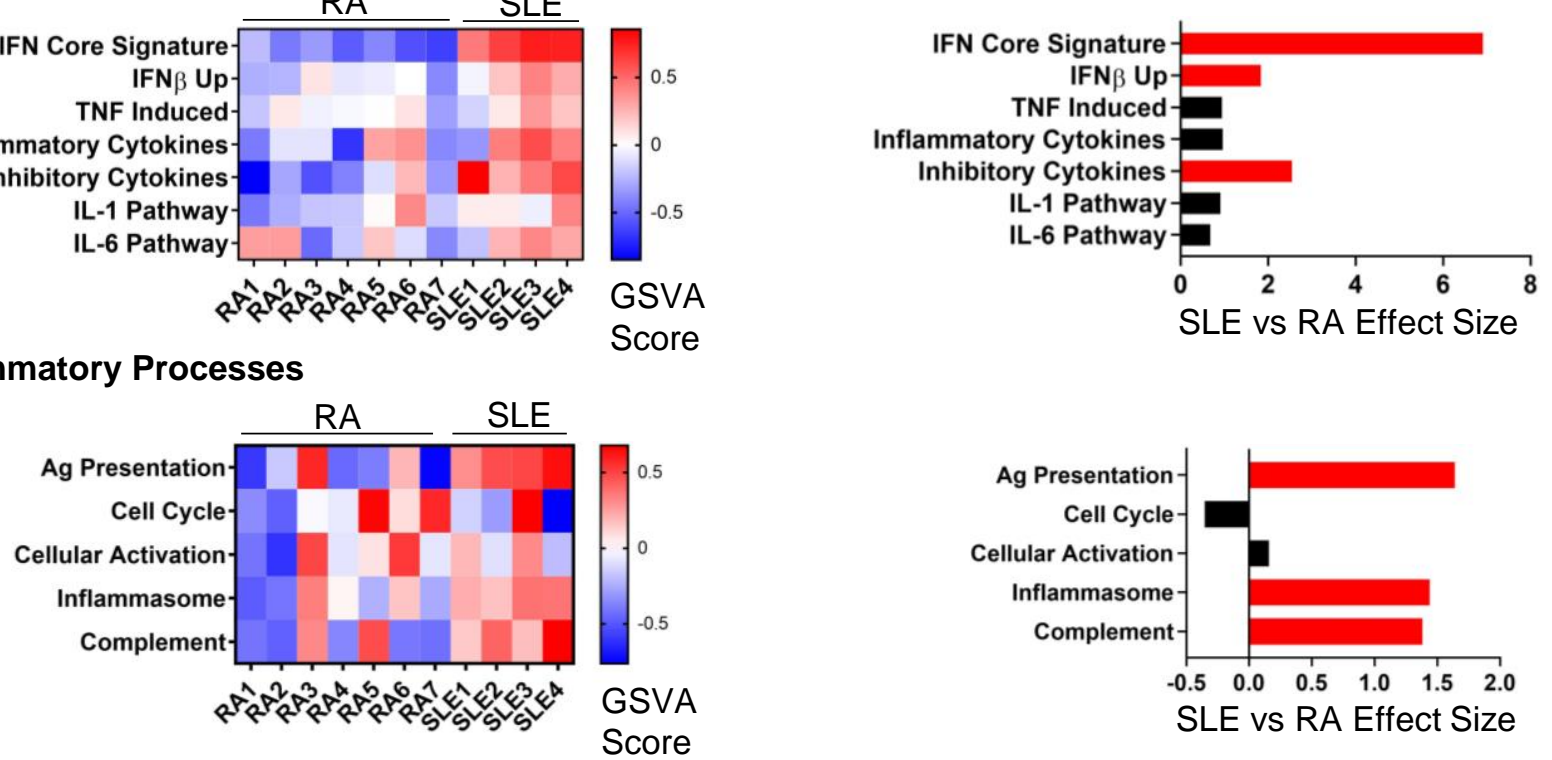

GSVA

Score

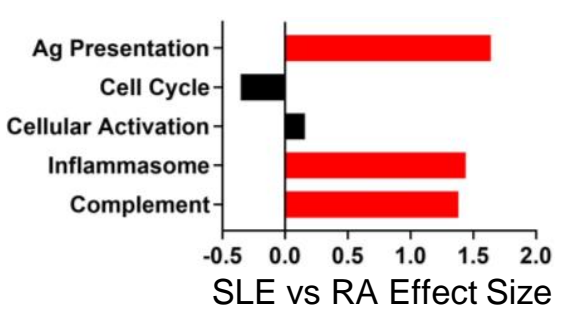

SLE vs RA Effect Size

Score
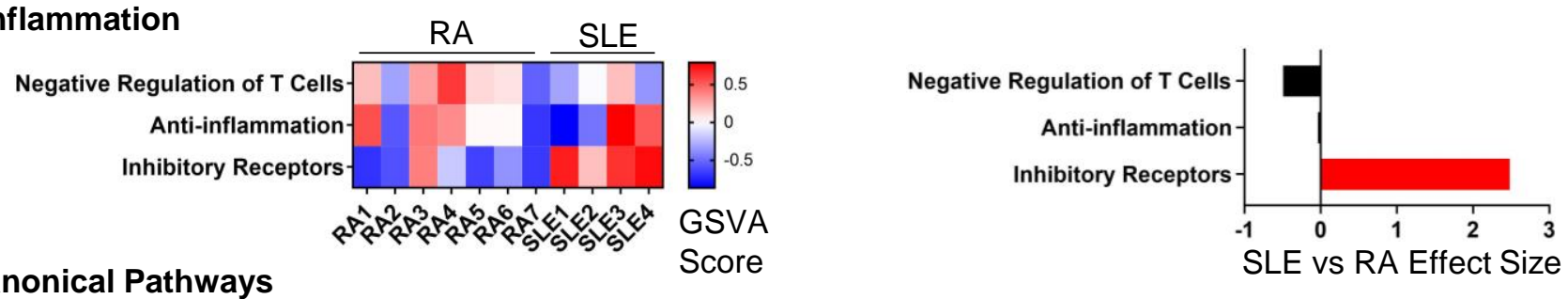

f IPA Canonical Pathways

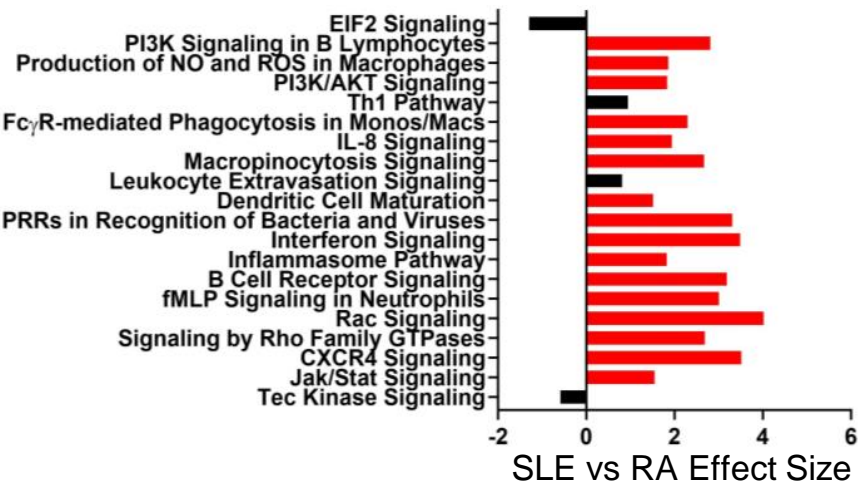

PI3K Signaling in B LymphalingPI3K Signaling in B Lymphocytes
Production of NO and ROS in Macrophages PI3K/AKT Signaling-

Fc $\gamma$ R-mediated Phagocytosis in Monos/MacsIL-8 Signaling Macropinocytosis Signaling. Leukocyte Extravasation Signaling RA SLE Role of PRRs in Recognition of Bacteria and Viruses. Interferon Signaling.

B Cell Receptor Signaling fMLP Signaling in Neutrophils Rac Signaling-
Signaling by Rho Family GTPases-
CXCR4 SignalingJak/Stat Signaling Tec Kinase Signaling 
bioRxiv preprint doi: https://doi.org/10.1101/2020.06.19.123307; this version posted June 21, 2020. The copyright holder for this preprint (which was not certified by peer review) is the author/funder. All rights reserved. No reuse allowed without permission.

$\mathbf{a}$

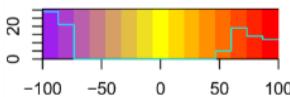

Connectivity Score

b

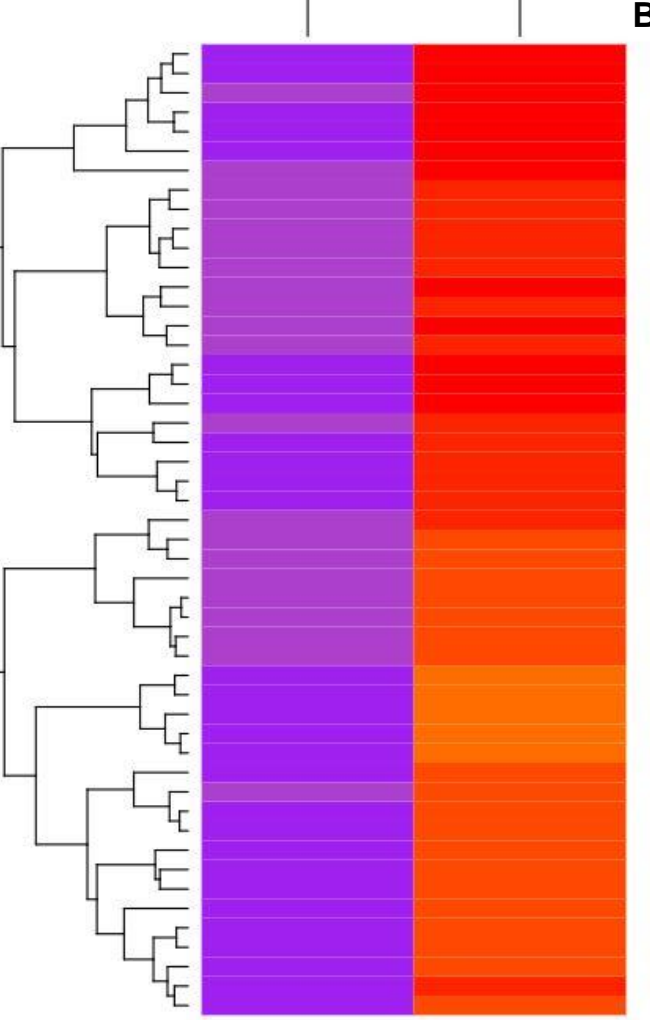

\section{LINCS \\ BUR}

IFNB1 - PF-06823859 55

TNFRSF1A Lauric acid $¥$

HNF4A $\longrightarrow$ Linoleic acid $¥$

ESR1 - Estrogen receptor antagonists

ELF4

IRF2

KLF6
ITGAE

CTBP2
HOXB13

OTX2

PASD1

MBNL1 \Ivachtin

FADD - PAC-1 1 PA

GCLM - Buthionine-sulfoximine ${ }^{\ddagger}$

GRK6 1 [Pkc-zeta inhibitor

RHOA - Y16P $\quad$ GF109203XP

CSK - go-6893

GABPB1 $\square$ Bosutinib ${ }^{\dagger}$

SF1 2 Apatinib‡

NFKBIB
SLC2A6 SRC-kinase-inhibitor-IP

ASGR2

PCGF3
VGLL4

CREB3L1

VDAC1

POU5F1

MYCBP Cholic acid

SOSTM1 Ubiquinol-1

DNAJB2 $\left\{\begin{array}{l}\text { Ubiquinol-1 } \\ \text { CObiquinones }\end{array}\right.$

BRD4 - Bromodomain inhibitors [Bumetanide ${ }^{\dagger}$

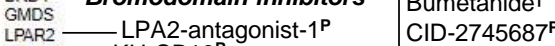

CLK3 $-\mathrm{KH}-\mathrm{CB} 19^{\mathrm{P}}$

$\mathrm{BRD} 3$ - Bromodomain inhibitors

CSNK1D-Casein kinase inhibitors

SNRNP70

Furosemide $^{\dagger}$

MLK

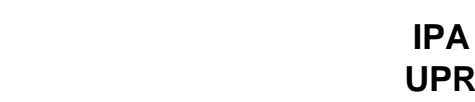
BI 655064

Iscalimab‡

FFP104 $\ddagger$ Dapirolizumab pegol ${ }^{\ddagger \overline{8}}{ }^{\mathrm{C}}$

Bleselumab $\ddagger$

NFKB pathway inhibitors [NFkB

PFI-3P $N$

GK921P

27219P Anifrolumab ${ }^{\ddagger 3}$

NFKB pathway inhibitors

JAK inhibitors

bitors -

JAK inhibitors MAP

Eritoran $¥$

Hydroxychloroquine ${ }^{+5}$ -

Resatorvid $\ddagger$ Hydroxychloroquine ${ }^{\dagger 5}{ }_{\text {TiC }}$ H

$$
\text { CU-CPT-4a } \mathrm{P} \text { DOCR }
$$

$$
\text { Dasa }
$$

PF-06823859 $¥ 5$ \{IFN IN N

$\mathrm{TwHF}^{+7}$

STA-5326

TIG

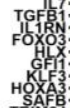

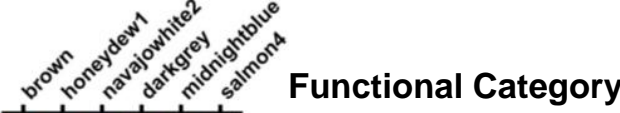

Chromatin Remodeling DNA Repair

- General Cell Surface

General Transcription

_ Glycolysis, Gluconeogen. \& Pentose Phosph. Pwys

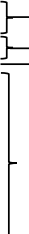

Immune Signaling

Interferon Stimulated Genes

Intracellular Signaling

Lysosome

MicroRNA Processing

MicroRNAs

Nucleus \& Nucleolus

Pattern Recognition

Receptors

Pro-Apoptosis

Proliferation

Secreted \& ECM

Daclizumab ${ }^{22}$

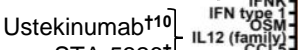

